\title{
Anisotropic strength behavior of single-crystal TATB
}

\author{
Matthew P. Kroonblawd, Brad A. Steele, Matthew D. Nelms, \\ Laurence E. Fried, and Ryan A. Austin \\ Lawrence Livermore National Laboratory, Livermore, CA 94550, United States \\ E-mail: kroonblawd1@llnl.gov
}

September 2021

\begin{abstract}
.
High-rate strength behavior plays an important role in the shock initiation of high explosives, with plastic deformation serving to localize heat into hot spots and as a mechanochemical means to enhance reactivity. Recent simulations predict that detonation-like shocks produce highly reactive nanoscale shear bands in the layered crystalline explosive TATB (1,3,5-triamino-2,4,6-trinitrobenzene), but the thresholds leading to this response are poorly understood. We utilize molecular dynamics (MD) to simulate the high-rate compressive stress-strain response of TATB, with a focus on understanding flow behavior. The dependence of strength on pressure and loading axis (crystal orientation) is explored. The deformation mechanisms fall broadly into two categories, with compression along crystal layers activating a buckling/twinning mode and compression normal to the layers producing nanoscale shear bands. Despite the complexity of the underlying mechanisms, the crystal exhibits relatively straightforward stress-strain curves. Most of the crystal orientations studied show rapid strain softening following the onset of yielding, which settles to a steady flow state. Trajectories are analyzed using five metrics for local states and structural order, but most of these metrics yield similar distributions for these deformation mechanisms. On the other hand, a recently proposed measure of intramolecular strain energy is found to most cleanly distinguish between these mechanisms, while also providing a plausible connection with mechanochemically accelerated decomposition kinetics. Localization of intramolecular strain energy is found to depend strongly on crystal orientation and pressure.
\end{abstract}

\section{Introduction}

Initiation of high explosives (HEs) involves a complicated hierarchy of physical and chemical processes over length scales ranging from Ångströms to centimeters $\left(10^{-10}\right.$ to $\left.10^{-2} \mathrm{~m}\right)$ and time scales ranging from femtoseconds to microseconds $\left(10^{-15}\right.$ to $\left.10^{-6} \mathrm{~s}\right)$. This interplay involves non-linear couplings between "fundamental" quantities and processes including equation of state (EOS), crystal mechanics, plastic deformation, phase transitions, transport, and chemical reaction kinetics. It is well understood 
that initiation stems from shock-induced hot spots that either form through plastic deformation in the bulk or when shock waves interact with microstructural defects such as pores or grain boundaries [1-3]. These hot spots are localized regions of elevated temperature/energy that accelerate chemistry and may produce self-sustained burn fronts that coalesce into a detonation [4]. Numerical simulations using multi-physics grain-scale models can predict initiation responses by explicitly resolving hot spot formation and evolution on its natural time and length scales (typically microns and nanoseconds), but these models are highly parameterized and require careful calibration of many physics terms [5-8]. Much of this fundamental physics remains elusive to direct experimental observation, especially at the extreme states (5-20 GPa, 500-1500 K) and small/fast scales (1-1000 ns, 0.001-10 mm) most relevant to the early stages of initiation. Sub-scale methods such as all-atom molecular dynamics (MD) are now viewed as indispensable tools to obtain these physics parameters [9-15], identify new underlying physicochemical processes [16-22], and directly validate grain-scale model predictions [23-25].

Mechanical processes contributing to initiation have historically been considered as sources for heat localization [2]. While shock compression leads to bulk heating through volumetric work governed by the unreacted EOS, this is insufficient to initiate most explosives. The dissipative work associated with deviatoric (strength) behavior is viewed as a significant contribution to heat localization through plastic deformation of bulk crystal and when shocks interact with pre-existing defects. Recent MD studies indicate that plastic work can enhance chemical reactivity in a more complicated way than simple localization of heat. Shearing in the bulk and at interfaces not only leads to heating [26-28], but can also enhance reaction kinetics and open new decomposition pathways in a range of explosives due to energy stored in molecular distortions [19,21,29]. Nanoscale shear bands are predicted to develop in bulk crystal under shock in many molecular explosives [21,30-33] and may contribute to ignition through a mechanochemical reduction of activation barriers to decomposition [21]. These nanoscale shear bands are distinct from conventional (adiabatic) shear bands that form at larger length scales due to thermal softening in regions of plastic deformation and instead arise due to intense shearing that stems from lattice breakdown/failure. Some of the first reactive MD simulations of shock-induced pore collapse predicted decomposition kinetics that were substantially faster than would be found at the same temperature and pressure in a perfect crystal [20]. Recently, excess intramolecular potential energy owing to deformation of individual molecules has been proposed as a plausible unifying concept linking these mechanochemical effects [22,34].

We focus here on assessing the strength behavior of TATB (1,3,5-triamino-2,4,6trinitrobenzene), which is an insensitive $\mathrm{HE}$ that is nearly unique in its safetyperformance characteristics. TATB is a triclinic molecular crystal [35] ( $P \overline{1}$ space group) and is comprised of layers of planar hydrogen-bonded molecules that stack in a graphitic-like motif (see Figure 1). Nanoscale shear banding is predicted in TATB under detonation-like shock conditions $(P=30 \mathrm{GPa})[21]$. Material in the bands was shown 
(a)

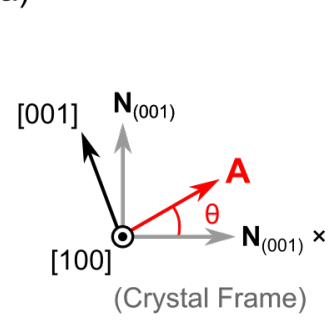

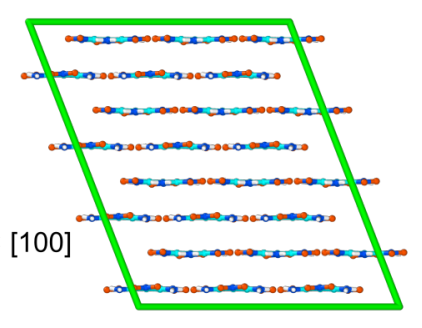

(b)

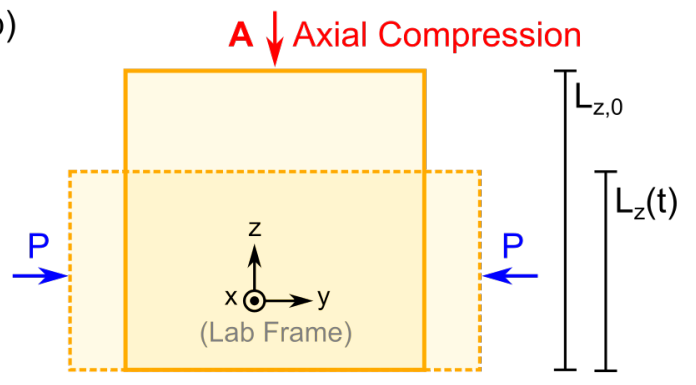

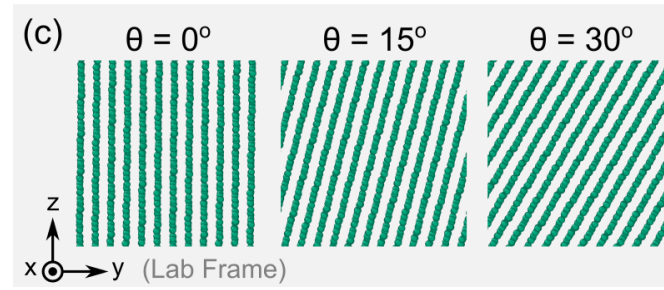

$\theta=45^{\circ}$
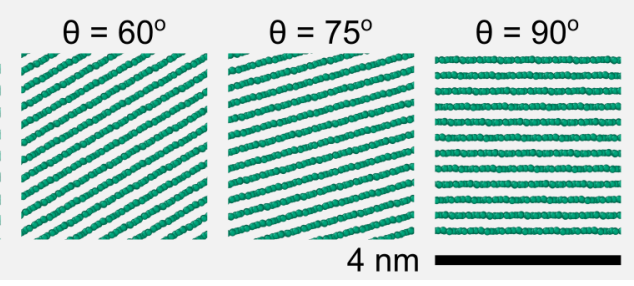

Figure 1. (a) Snapshot of a $3 \times 3 \times 4$ TATB crystal supercell and definitions for axial compression vector $\mathbf{A}$ in relation to crystal lattice directions. (b) Schematic initial-boundary value problem for axial compression simulations in the Cartesian lab frame. (c) Snapshots of the seven crystal orientations considered for axial compression simulations denoted by angle $\theta$.

to react $\approx 200 \times$ faster than material outside the bands at typical shock temperatures, offering a qualitative explanation for unusual changes in chemical reactivity that vary parametrically with shock pressure [36-39]. Up-scaling this physics to higher-level models requires understanding strength behavior under large strains and high strain rates.

Most of the available information on elastic and inelastic deformations in TATB crystal has been determined within the past decade through MD simulations. This is due to the poor quality and brittleness of most lab-grown crystals [40], which has until recently prevented experiments on large high-quality single crystals [41,42]. The full set of elastic coefficients was first determined in 2009, with the development and application of what is now a widely used TATB force field (FF) model by Bedrov et al. [43]. One of the dominant features of the elastic response is that the crystal is substantially more compliant normal to the layers than within the layers. Glide of the layers within the (001) basal plane is particularly facile, with stacking fault energies that are similar to graphite $[44,45]$; such events are readily observed in MD simulations [46,47]. The single high-pressure monoclinic polymorph of TATB is also layered and related to the ambient triclinic form though a set of layer displacements [41].

Lafourcade et al. [48] recently identified a number of deformation modes in addition to basal glide. This includes a buckling/twinning mode that is observed when the compression axis falls approximately within the basal plane and transverse slip with dislocation motion that involves breaking the molecular layers. Many of these mechanisms are predicted to operate in single crystals under shock conditions, but nanoscale shear banding can also arise $[21,49]$. Direct shock simulations by Zhao et 
al. [49] probing the orientation-dependent response near $10 \mathrm{GPa}$ show that nucleation of inelastic deformations is prompt, occurring on timescales of 10 s of picoseconds or less. The contact mechanics exhibit anisotropy, with MD-simulated nanoindentation predicting a hardness of $\approx 1 \mathrm{GPa}$ for the (001) basal plane and non-Hertizan responses for the (100) and (010) planes that expose the molecular layers [50]. Recent nanoindentation experiments probing the response normal to the (001) plane yielded a hardness that was somewhat smaller than MD predictions (0.41 GPa), a Young's modulus that was the same as MD predictions within uncertainty, and an average shear stress at yield of $0.38 \mathrm{GPa}[51]$.

The above studies have revealed many details regarding TATB crystal mechanics, but significant gaps remain that leave uncertainty in how to best model strength effects at shock initiation and detonation conditions. While pressure-dependent nucleation stresses are well-characterized $[45,48,52]$, these measures focus on the conditions of incipient plasticity and transients associated with the sudden loading of perfect crystal. For models that seek to capture multi-crystal response at longer time scales, it is essential to understand the flow behavior at large strains.

Quantifying deformations in MD trajectories of complicated materials such as TATB is made difficult due to the low symmetry class (triclinic) and highly varied mechanisms that have thus far been identified. Most established atomic-scale order parameters and structural metrics are focused on specific types of defects in cubic crystals (e.g., metals) [53-55]. Previous shock studies on molecular explosives have used molecular orientation vectors, tailored conformational measures, and near-neighbor displacement maps (among others) to measure local order [22,30,32,33,49]. Definitions of the local strain tensor and shear strain are also available [56,57], but combine all deviatoric components and are formally defined for affine deformations only. Using intramolecular potential energy as a measure of intramolecular strain energy may be a more universal metric and is anticipated to closely connect to chemistry [22].

We focus here on determining the orientation- and pressure-dependent strength behavior of TATB as a basis for developing a grain-scale model for TATB with "strength-aware" chemical kinetics. The approach combines MD simulations of uniaxial compressive stress-strain loading with oriented samples prepared using the generalized crystal-cutting method (GCCM) [58]. Through a series of large-scale MD simulations, we first determine the basic features of TATB stress-strain response beyond the onset of yielding for a range of crystal orientations and pressures. The underlying trajectories are then analyzed in greater detail using five metrics sensitive to different aspects of the crystal deformations. These metrics reveal mechanisms responsible for specific stressstrain responses and provide a route to identify and quantify highly sheared material, which is relevant to advanced chemistry models that account for shear band ignition [39]. 


\section{Methods}

\subsection{Force Field Model and Trajectory Integration}

Classical all-atom molecular dynamics (MD) simulations were performed using LAMMPS [59], 3D-periodic simulation cells, and a variant of the well-established non-reactive force field (FF) for TATB developed by Bedrov et al. [43]. The variant of the TATB FF used here incorporates tuned harmonic bond and angle potentials [60] and a repulsive intramolecular potential between $\mathrm{O}$ and $\mathrm{H}$ atoms [61]. Non-bonded terms were evaluated in real space up to an $11 \AA$ cutoff and the electrostatic terms were evaluated using the Wolf potential [62] with an 11 A cutoff. Full descriptions of the TATB FF and LAMMPS implementation details can be found in recent reports [23,49]. A Nosé-Hoover style thermostat $[63,64]$ and barostat [65] with respective coupling constants of $100 \mathrm{fs}$ and 1000 fs were used at various points in this study and are discussed for each application below. All trajectories were integrated using a 0.5 fs timestep. Renderings of the trajectories were prepared using OVITO [66].

The simulations involve uniaxial compression of a single crystal held at a prescribed pressure and temperature. This is analogous to the simple compression measurements used to determine stress-strain curves, and similar to the MD protocol described in Ref. [67]. The same lab coordinate frame was used for all compression simulations and is given in Figure $1(\mathrm{~b})$. Here, a quasi-2D simulation cell with approximate $x y z$ dimensions $8 \mathrm{~nm} \times 100 \mathrm{~nm} \times 100 \mathrm{~nm}$ is axially compressed along $z$ at constant true strain rate $\dot{\epsilon}_{z z}$ through scaling the $L_{z}(t)$ cell length in time $t$ according to

$$
\epsilon_{z z}=\ln \frac{L_{z}(t)}{L_{z, 0}}=\dot{\epsilon}_{z z} \cdot t .
$$

Here, $\epsilon_{z z}$ is the true strain and $L_{z, 0}$ is the starting length of the cell along $z$ prior to compression. Dynamic strains were implemented in LAMMPS though a "fix deform" command in which the deformation was incremented every time step and the atomic positions were remapped affinely. An approximately uniform background pressure was maintained through independently coupled barostats in the transverse directions. A thermostat with target temperature of $300 \mathrm{~K}$ was applied during the compression to offset large temperature rises due to dissipative work. Note that while these choices yield a convenient initial-boundary value problem, the resulting trajectories are fundamentally nonequilibrium and do not conform to a formal thermodynamic ensemble.

The compression response was determined for seven TATB crystal orientations and seven distinct pressure states $P \in[0 \mathrm{GPa}, 30 \mathrm{GPa}]$, corresponding to a total of 49 distinct cases. The specific pressures considered are 1 atm (hereafter denoted as $0 \mathrm{GPa}$ ) and elevated pressures of 1, 3, 5, 10, 20, and $30 \mathrm{GPa}$ that span application conditions ranging from room pressure up to steady detonation. The axial compression vector $\mathbf{A}$ is shown in Figure 1(a) and (b) in relation to the lab frame and crystal frame defined by non-orthogonal lattice vectors $\mathbf{a}=[100], \mathbf{b}=[010]$, and $\mathbf{c}=[001]$. This compression vector is defined in terms of angle $\theta$, which is the complement of the polar angle measured 

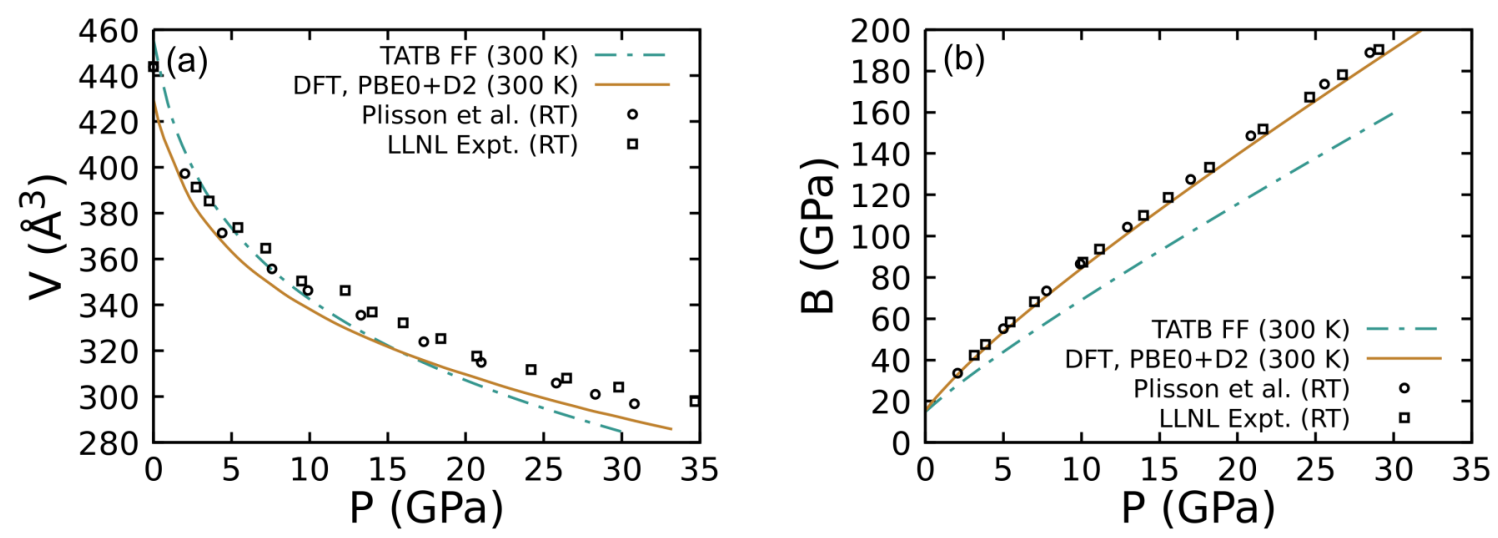

Figure 2. (a) Pressure-volume response for two molecules in TATB crystal, which is equivalent to the unit cell volume for the $P \overline{1}$ phase. (b) Bulk modulus as a function of pressure determined from fits of the data in panel (a) to a Birch-Murnaghan EOS.

with respect to the TATB basal plane normal vector $\mathbf{N}_{(001)}=[100] \times[010]$. Vector $\mathbf{A}$ lies in the plane defined by the vectors $\mathbf{N}_{(001)} \times[100]$ and $\mathbf{N}_{(001)}$, which leads to lattice vector [100] coinciding with $x$ in the lab frame for all 49 simulations. Thus defined, the vector $\mathbf{A}$ spans cases bounded by compression exactly within the crystal layers $\left(\theta=0^{\circ}\right)$ and compression exactly normal to the crystal layers $\left(\theta=90^{\circ}\right)$. Large-strain behavior may depend on the azimuthal angle of rotation about $\mathbf{N}_{(001)}$, but is not considered here. Earlier work [48] showed that incipient plasticity was approximately independent of this azimuthal angle.

Starting configurations for the 49 simulations were prepared using lattice parameters determined from isothermal-isobaric $(N P T)$ simulations and the generalized crystal-cutting method (GCCM) [58]. The NPT simulations were performed at $300 \mathrm{~K}$ using an $8 \times 8 \times 12$ supercell of the $P \overline{1}$ phase [35] and a fully triclinic barostat in which the six stress components were independently coupled to their respective cell parameters. Each NPT simulation was $1000 \mathrm{ps}$ long and the lattice parameters were obtained as averages over the last 500 ps. Based on these lattice parameters, the GCCM was used to construct nearly orthorhombic simulation cells for the 49 cases subject to the target dimensions and geometric definitions given above. The resulting simulation cells contained between $\approx 8$ and $\approx 15$ million atoms. Each configuration was thermalized at $300 \mathrm{~K}$ through a $25 \mathrm{ps}$ isothermal-isochoric $(N V T)$ simulation prior to axial compression. Thus, the initial state for each compression simulation was hydrostatic with a pressure equal to the target pressure of the transverse barostats.

While the TATB FF has been applied in shock simulations reaching $\approx 30 \mathrm{GPa}$, to our knowledge this is the first application of the fully flexible TATB FF in isobaric simulations above $10 \mathrm{GPa}$. The highest pressures considered involve extrapolation of the TATB FF to states beyond the initial fitting regime. As a point of comparison, Figure 2(a) shows the present predictions for the $300 \mathrm{~K} P(V)$ isotherm determined with the TATB FF to the "best available" quantum chemistry predictions [47] and recent 
Table 1. Fitted Birch-Murnaghan parameters for the TATB $300 \mathrm{~K}$ isotherm.

\begin{tabular}{llll}
\hline Data Set & $V_{0}\left(\AA^{3}\right)$ & $B_{0}(\mathrm{GPa})$ & $B_{0}^{\prime}$ (unitless) \\
\hline TATB FF $(300 \mathrm{~K})$ & 449.418 & 14.8927 & 6.90624 \\
DFT, PBE0+D2 $(300 \mathrm{~K})$ & 426.334 & 15.1278 & 10.4692 \\
Plisson et al. (RT) & 433.893 & 14.5611 & 11.5721 \\
LLNL Expt. (RT) & 437.762 & 16.1325 & 10.6891 \\
\hline
\end{tabular}

experiments $[47,68,69]$. The quantum chemistry results were obtained through density functional theory (DFT) calculations of the $P \overline{1}$ phase at the PBE0 level of theory [70] and include D2 dispersion corrections [71], zero-point energy, and thermal effects evaluated within the quasi-harmonic approximation at $300 \mathrm{~K}$. Both sets of experiments were performed using diamond anvil cells at room temperature (RT). The results from Plisson et al. [68] were indexed to the $P \overline{1}$ phase for the entire pressure interval. Data from LLNL experiments includes separate determinations below [69] and above [47] the subtle phase transition [41] to $I 2 / a$ at $\approx 4 \mathrm{GPa}$, with points above the phase transition pressure being indexed to $I 2 / a$. The four sets of data were independently fit to a $3^{\text {rd }}$ order Birch-Murnaghan EOS form for the $300 \mathrm{~K}$ isotherm,

$$
P(V)=\frac{3 B_{0}}{2}\left[\left(\frac{V_{0}}{V}\right)^{7 / 3}-\left(\frac{V_{0}}{V}\right)^{5 / 3}\right]\left\{1+\frac{3}{4}\left(B_{0}^{\prime}-4\right)\left[\left(\frac{V_{0}}{V}\right)^{2 / 3}-1\right]\right\},
$$

which is defined in terms of a reference unit cell volume $V_{0}$, the bulk modulus at the reference $B_{0}$, and its pressure derivative $B_{0}^{\prime}$. Least-squares fits of Equation 2 to the four data sets were obtained for pressures $P \in[0 \mathrm{GPa}, 30 \mathrm{GPa}]$ treating $V_{0}, B_{0}$, and $B_{0}^{\prime}$ as free parameters. These fitted parameters are collected in Table 1 and plots of the pressure-dependent bulk modulus obtained via the partial derivative with respect to volume are shown in Figure 2(b).

Several trends are apparent from comparison of the isotherm data. The TATB FF closely matches experimental determinations of the unit cell volume between $0 \mathrm{GPa}$ and $\approx 10 \mathrm{GPa}$, but is too compliant at higher pressures. This is perhaps not surprising as the TATB FF was originally validated against the diamond anvil cell experiments of Stevens et al. [72], which went up to $13 \mathrm{GPa}$ and were the best-available data at the time. In comparison, the DFT results consistently underestimate the experimental volume, but do so essentially by a constant value, yielding a similar shaped curve. These differences manifest clearly in the pressure-dependent bulk modulus. Excess compliance of the TATB FF is apparent, differing from experiment by $\approx 20 \%$ percent at $30 \mathrm{GPa}$, while the DFT results are practically the same as experiment. Despite these results, the TATB FF is nonetheless a reasonable model for the present application, which requires long-timescale simulations involving many millions of atoms. Re-parameterization to improve the high-pressure EOS response of the TATB FF is the subject of future work. 


\subsection{Trajectory Analysis}

2.2.1. Global Response Global properties were computed over all atoms in the system during each axial compression simulation, including the simulation cell geometry and LAMMPS-computed stress tensor $\mathbf{P}$, which is interpreted as the Cauchy stress (positive under compression).

The LAMMPS simulation cell is defined in terms of three cell vectors $\mathbf{x}_{1}, \mathbf{x}_{2}$, and $\mathbf{x}_{3}$, which are the columns of the matrix

$$
\mathbf{H}=\left(\begin{array}{lll}
\mathbf{x}_{1} & \mathbf{x}_{2} & \mathbf{x}_{3}
\end{array}\right)=\left(\begin{array}{ccc}
L_{x} & x y & x z \\
0 & L_{y} & y z \\
0 & 0 & L_{z}
\end{array}\right)
$$

In the axial compression simulations, barostats are independently coupled to the first two vector lengths $\left(\left|\mathbf{x}_{1}\right|\right.$ and $\left.\left|\mathbf{x}_{2}\right|\right)$ to maintain a constant stress in those directions while a strain is imposed on the $z$ component of the third vector given by Equation 1 . The angle between $\mathbf{x}_{1}$ and $\mathbf{x}_{2}$ is a constant, so the value $x y$ changes in time. The $x z$ and $y z$ components of $\mathbf{x}_{3}$ are constant. The deformation gradient tensor

$$
\mathbf{F} \approx \mathbf{H} \cdot \mathbf{H}_{0}^{-1}
$$

is computed from the instantaneous $\mathbf{H}$ and its reference value $\mathbf{H}_{0}$ at the start of the simulation. Note that Eq. 4 approximates the true deformation gradient because material can move independent of the simulation cell despite the molecule positions being affinely remapped, but this approximation is reasonable for elastic portions of the compression.

For a given loading direction $\mathbf{A}$, we compute an effective shear modulus by considering the change in angle between various sets of orthogonal line elements $\left(\mathbf{n}_{\mathbf{0}}, \mathbf{s}_{\mathbf{0}}\right)$ during a small elastic deformation. As shown in Figure 3, the line elements are oriented $\pm 45^{\circ}$ away from the loading axis in the reference frame. We define an effective shear modulus by averaging with respect to line element rotations about the loading axis, i.e.,

$$
G_{\text {eff }} \equiv\left\langle\frac{\tau}{\gamma}\right\rangle_{\mathbf{A}}
$$

where $\tau$ and $\gamma$ are, respectively, the projected shear stress and shear strain on the system defined by $\left(\mathbf{n}_{\mathbf{0}}, \mathbf{s}_{\mathbf{0}}\right)$. In this averaging scheme, the crystal is held fixed as the line element pair is rotated and, in this setting, the compression axis $\mathbf{A}$ is always aligned with the lab frame $z$-axis. The projected shear stress is obtained as

$$
\tau=\mathbf{n}_{0} \cdot \mathbf{S} \cdot \mathbf{s}_{0}
$$

where the second Piola-Kirchhoff stress $\mathbf{S}$ is expressed as

$$
\mathbf{S}=(\operatorname{det} \mathbf{F}) \mathbf{F}^{-1} \cdot \mathbf{P} \cdot \mathbf{F}^{-\mathrm{T}} .
$$

The shear strain is obtained as

$$
\gamma=\frac{\pi}{2}-\zeta
$$

where $\zeta$ is determined by the corresponding vectors mapped into the current frame $\left(\mathbf{n}=\mathbf{F} \cdot \mathbf{n}_{0}, \mathbf{s}=\mathbf{F} \cdot \mathbf{s}_{0}\right)$. 


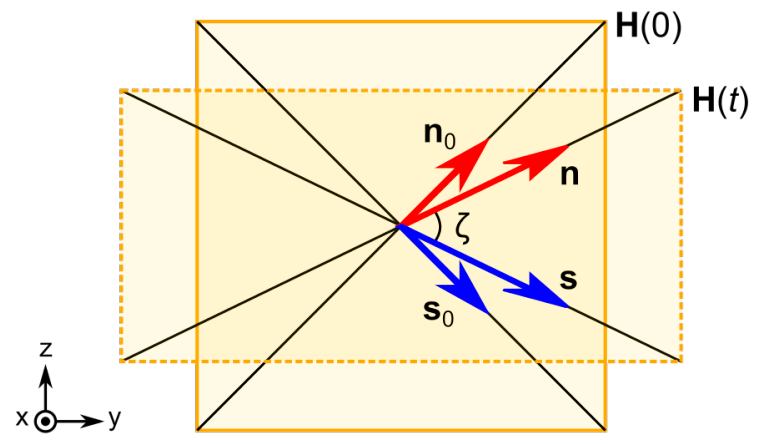

Figure 3. Schematic for evaluating the shear stress and strain for small strains.

2.2.2. Local Response Inelastic deformation mechanisms were characterized through analysis of the all-atom trajectories using five local metrics to characterize deformation response. These metrics include the temperature, the potential energy associated with molecular deformation (an intramolecular strain energy), two structural parameters sensitive to rotational order, and an atomic measure of shear strain. We focus here on definitions for these metrics and reserve discussion on the advantages, disadvantages, and interpretations of these metrics in the context of specific examples for Section 3.2.

The general work flow consists of two parts. In the first step we determine properties of whole molecules from the underlying atomic quantities including positions, velocities, and potential energies. In the second step, averaging and evaluations over a molecule's local neighborhood are performed within a sphere with cutoff radius $r_{0}=1.5 \mathrm{~nm}$ about a given molecule center of mass (COM). This second operation serves to smooth fluctuations and is necessary for the evaluation of some order parameters.

Starting from atomic positions $\mathbf{r}_{i}$, velocities $\mathbf{v}_{i}$, and intramolecular potential energies $p e_{\text {intra, } i}$, we compute the molecular COM position, total kinetic energy, and total intramolecular potential energy as

$$
\mathbf{R}=\frac{1}{M} \sum m_{i} \mathbf{r}_{i}
$$

and

$$
K E_{\mathrm{tot}}=\sum \frac{1}{2} m_{i} \mathbf{v}_{i} \cdot \mathbf{v}_{i}
$$

and

$$
P E_{\text {intra }}=\sum p e_{\text {intra }, i}
$$

Each sum runs over the 24 atoms in a TATB molecule, $m_{i}$ is the mass of atom $i$, and $M$ is the mass of a molecule. The per-atom intramolecular potential energy is computed from the bond, angle, dihedral, and improper dihedral terms in the TATB FF. The kinetic energy is directly proportional to the temperature, which is computed as

$$
T=\frac{2 K E_{\mathrm{tot}}}{72 k_{\mathrm{B}}}
$$

where $k_{\mathrm{B}}$ is the Boltzmann constant and the factor of 72 arises from the number of degrees of freedom in a TATB molecule. Based on the atom positions and a consistent 
(a)

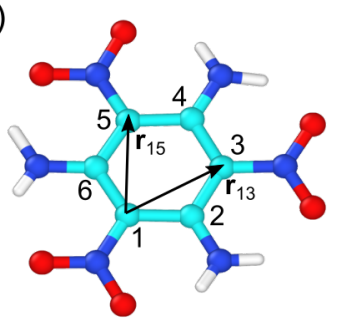

(b)

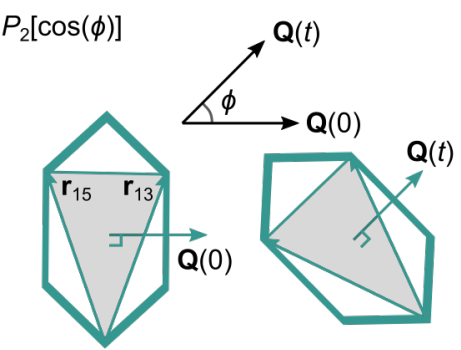

(c) Rotational

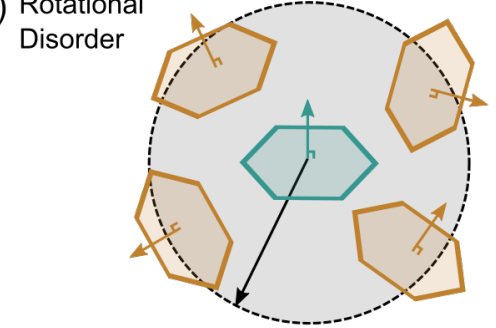

Figure 4. (a) TATB molecule and numbering scheme used to define separation vectors $\mathbf{r}_{13}$ and $\mathbf{r}_{15}$. (b) Definition of the $P_{2}[\cos (\phi)]$ order parameter in terms of timedependent changes in the molecular normal vector $\mathbf{Q}(t)$. (c) Local rotational disorder is characterized by computing the alignment of molecular normal vectors within the local neighborhood defined by a sphere centered on each molecule COM.

atom numbering scheme given in Figure 4(a), we also compute the unit normal vector for each molecule as

$$
\mathbf{Q}=\frac{1}{2}\left[\mathbf{r}_{15} \widehat{\times} \mathbf{r}_{13}+\mathbf{r}_{26} \widehat{\times} \mathbf{r}_{24}\right]
$$

where $\hat{\mathbf{q}}$ is the unit vector obtained by normalization of $\mathbf{q}$. A final normalization was applied to $\mathbf{Q}$ prior to subsequent analysis as the definition for $\mathbf{Q}$ does not necessarily yield a unit vector.

The first local metric, the temperature, can be directly obtained through local averaging about each molecule using molecular temperatures computed with Equation 12. The remaining four metrics require further manipulations as they are either defined with respect to a reference state and/or involve evaluations of local geometry.

The second metric we consider is a form of intramolecular strain energy associated with deformation of molecular shape, first defined in Ref. [22]. Equipartition of energy amongst quasi-harmonic vibrational modes predicts that a change in temperature will lead to equal changes in kinetic and potential energy. Thus, the difference between changes in energy gives insight into changes in phase. We isolate the energy associated with strain of molecule shape in terms of the above molecular quantities as

$$
U_{\text {intra }}=\left[P E_{\text {intra }}-P E_{\text {intra }, 0}\right]-\frac{66}{2} k_{\mathrm{B}}[T-300 \mathrm{~K}]
$$

Here, the first term in brackets is the change in intramolecular potential energy with respect to the start of an axial compression simulation while the second term is the change in kinetic energy associated with the 66 internal (vibrational) degrees of freedom. It is worth noting that the potential energy term dominates the above expression for the simulations reported here, with the kinetic term serving as a minor correction for inhomogeneity of the temperature field.

The third local metric, and the first which explicitly measures structure, is expressed in terms of the second Legendre polynomial of a molecular orientation cosine

$$
P_{2}[\cos (\phi)]=\frac{1}{2}\left[3 \cos ^{2}(\phi)-1\right] .
$$


This metric has been previously employed to analyze MD shock simulations $[21,31,32]$ and considers only how the orientation of a vector attached to a molecule changes in time as measured with respect to a single reference configuration. Following the schematic in Figure 4(b), we take the molecular normal vector $\mathbf{Q}(t)$ to define $\cos (\phi)$ where $\mathbf{Q}(0)$ corresponds to the start of axial compression. The lower and upper bounds for $P_{2}[\cos (\phi)]$ are -0.5 and 1.0 , with the latter corresponding to $\phi=0$ and thus no net change in molecular orientation.

The fourth local metric also measures rotational order based on molecular normal vectors $\mathbf{Q}(t)$, but is distinct from $P_{2}[\cos (\phi)]$. For a given pair of molecules $i$ and $j$ at a point in time $t$, we compute their relative alignment angle as

$$
\psi_{i j}(t)=\arccos \left|\mathbf{Q}_{i}(t) \cdot \mathbf{Q}_{j}(t)\right|,
$$

which is evaluated from an unsigned dot product between molecular unit normal vectors. Averaging about molecule $i$ 's local neighborhood as in Figure 4(c) yields a measure of local rotational (dis)order,

$$
\langle\psi\rangle=\frac{1}{N_{i}} \sum_{j, r_{i j} \leq r_{0}} \psi_{i j}(t)
$$

where $N_{i}$ is the number of molecules whose COM is within $r_{0}=1.5 \mathrm{~nm}$ of molecule $i$. Thus defined, this measure of local rotational disorder depends only on the instantaneous configuration rather than a reference state. It is bounded between $0^{\circ}$ and $90^{\circ}$, with the former indicating that all molecules are locally aligned in the same direction.

The fifth and final metric we consider is a local measure of shear strain $\epsilon_{\mathrm{II}}$, which is derived from the local Green-Lagrange strain tensor,

$$
\mathbf{E}=\frac{1}{2}\left(\mathbf{F}^{\mathrm{T}} \cdot \mathbf{F}-\mathbf{I}\right)
$$

Here, $\mathbf{F}$ is the local deformation gradient tensor and $\mathbf{I}$ is the identity tensor. To compute F, we follow the method of Refs. [56] and [57] as implemented in OVITO [66] and operate on molecular COM positions. The local deformation gradient about molecule $i$ is computed in matrix notation with respect to a reference configuration as

$$
\mathbf{F}_{i}=\sum_{j, r_{i j} \leq r_{0}} \mathbf{x}_{i j} \mathbf{X}_{i j}^{\mathrm{T}}\left(\sum_{j, r_{i j} \leq r_{0}} \mathbf{X}_{i j} \mathbf{X}_{i j}^{\mathrm{T}}\right)^{-1} .
$$

Here, $\mathbf{x}_{i j}$ denotes the separation vector between molecule $i$ and $j$ in the current configuration and similarly $\mathbf{X}_{i j}$ for the reference configuration, taken to be the frame from the start of axial compression. Quantities $\mathbf{Y}$ and $\mathbf{Y}^{\mathrm{T}}$ respectively denote column and row vectors, such that their product as written is a $3 \times 3$ matrix. The shear strain scales with the second invariant of $\mathbf{E}$ and is evaluated from the six independent $E_{i j}$ components as

$$
\begin{aligned}
& \epsilon_{\mathrm{II}}=\left[\left(E_{11}-E_{22}\right)^{2}+\left(E_{11}-E_{33}\right)^{2}+\left(E_{22}-E_{33}\right)^{2}\right. \\
& \left.+\frac{1}{6}\left(E_{12}^{2}+E_{13}^{2}+E_{23}^{2}\right)\right]^{1 / 2} .
\end{aligned}
$$



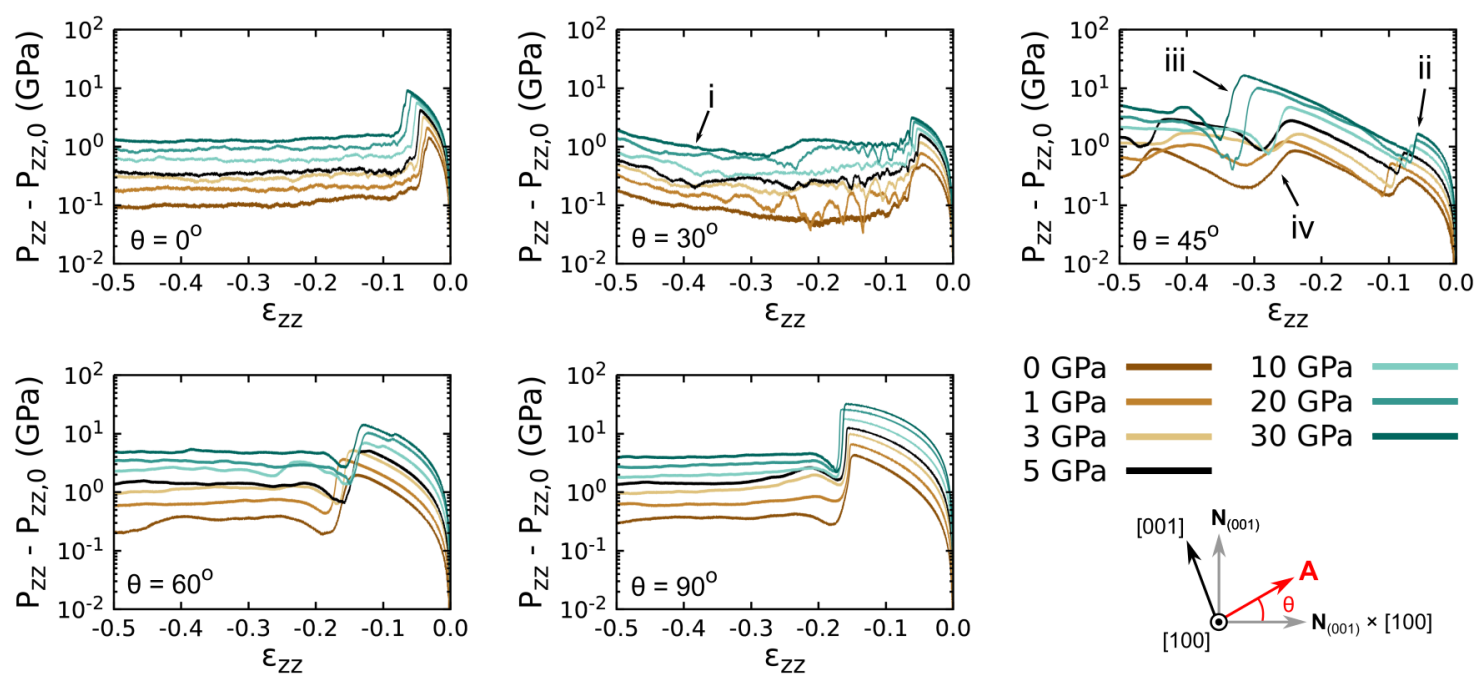

Figure 5. Stress-strain curves for axial compression of selected TATB crystal orientations denoted by $\theta$ in each panel at rate $\dot{\epsilon}_{z z}=-1.0 \times 10^{9} \mathrm{~s}^{-1}$. Each curve corresponds to an independent simulation at the specified background pressure. Note that axial stress is plotted on a logarithmic scale

The shear strain thus defined is based on changes of local packing environment with respect to a reference configuration within the assumption of affine deformations. As will be discussed in Section 3.2, these considerations complicate its application to TATB deformations under large strains.

\section{Results and Discussion}

\subsection{Stress-Strain Response}

The stress-strain response of TATB crystal was predicted for seven different crystal orientations $\theta \in\left[0^{\circ}, 90^{\circ}\right]$ and seven distinct pressure states $P \in[0 \mathrm{GPa}, 30 \mathrm{GPa}]$ following the approach outlined in Section 2.1. Each of the 49 simulations was performed with a true strain rate of $\dot{\epsilon}_{z z}=-1.0 \times 10^{9} \mathrm{~s}^{-1}$ and a final true strain of $\epsilon_{z z}=-0.5$, which corresponds to 500 ps. This rate is of a similar magnitude to those encountered in ultrafast shock experiments [73]. While characterization of rate effects is a subject of ongoing work, we have found essentially no rate dependence in the $\left(\theta=90^{\circ}, P=3 \mathrm{GPa}\right)$ and $\left(\theta=90^{\circ}, P=30 \mathrm{GPa}\right)$ cases for compression rates $\left|\dot{\epsilon}_{z z}\right| \in\left[5.0 \times 10^{7} \mathrm{~s}^{-1}, 1.0 \times 10^{9} \mathrm{~s}^{-1}\right]$. Representative stress-strain curves are shown in Figure 5, with each panel corresponding to a different orientation. The axial stress $P_{z z}-P_{z z, 0}$ is measured with respect to the reference pressure $P_{z z, 0}$ in each case. Responses for $\theta=15^{\circ}$ and $75^{\circ}$ are not shown as they are qualitatively similar to $0^{\circ}$ and $90^{\circ}$, respectively.

There are several qualitative features that are largely consistent across most of the cases. The first is an approximately linear increase in stress at small strains, followed by sudden yielding (onset of plastic flow) and softening in each stress-strain curve. Following the maximum, the flow stress tends to settle down to an approximately 

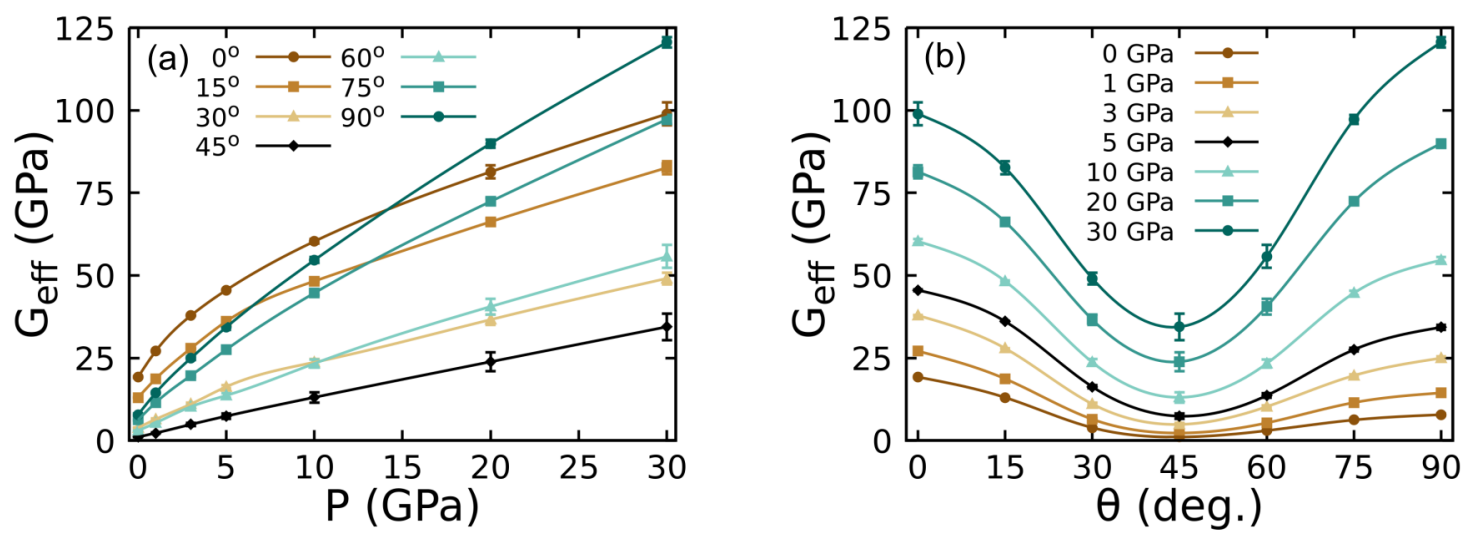

Figure 6. Effective shear modulus plotted as (a) a function of pressure, and (b) a function of crystal orientation. Curves correspond to spline fits.

constant value, which depends on pressure and orientation. Notable exceptions to the above behavior are highlighted by roman numerals in the $30^{\circ}$ and $45^{\circ}$ cases of Figure 5 . For example, the $30^{\circ}$ case at high pressure exhibits apparent hardening at large strain $\epsilon_{z z} \leq-0.4$ denoted by (i). The response at $45^{\circ}$ is even more complicated, including an initial relaxation at smaller strain (ii), and differences in relaxation characteristics at larger strains seen for high (iii) and low (iv) pressure. A similar low-strain relaxation is also seen in the $60^{\circ}$ case for pressures above $5 \mathrm{GPa}$. These features can be traced to specific mechanisms, which will be discussed in Section 3.2.

General characteristics of the stress-strain response are phenomenologically similar to glassy polymers, which often exhibit an initial elastic-anelastic response, followed by a yielding peak, rapid strain softening, and a long-tailed plateau stress corresponding to steady plastic flow [74]. Metallic perfect crystals (without seeded dislocations) can also behave similarly under these high strain rates [67]. However, unlike metals, there is currently no experimental evidence of pre-existing dislocations in TATB crystals. Labgrown TATB crystals are typically of poor quality [40] unless carefully prepared $[41,42]$ and known to contain nanoscopic voids [75]. Our preliminary MD simulations with $\theta=0^{\circ}$ and $90^{\circ}$ oriented crystals at $0 \mathrm{GPa}$ containing cylindrical voids (not shown) indicate that such voids lower the initial yield point, but do not appreciably alter the average steady flow stress.

Effective shear moduli $G_{\text {eff }}$ were extracted from the axial compression simulations using Equation 5 at shear strain $\gamma=0.025$. Each data point is an average for rotation about the loading axis and error bars are taken as the standard deviation. These moduli are plotted as functions of pressure and crystal orientation in Figure 6. It is clear that the effective shear modulus for various loading directions is highly anisotropic, especially at high pressure where it exhibits a spread of $\approx 86 \mathrm{GPa}$. There is a minimum for each pressure at $\theta=45^{\circ}$, which corresponds to a larger shear stress along the TATB crystal layers. The orientation for the global maximum shifts from $\theta=0^{\circ}$ at low pressure to $\theta=90^{\circ}$ at high pressure. Inspection of the interpolation splines in Figure 6(a) indicates 


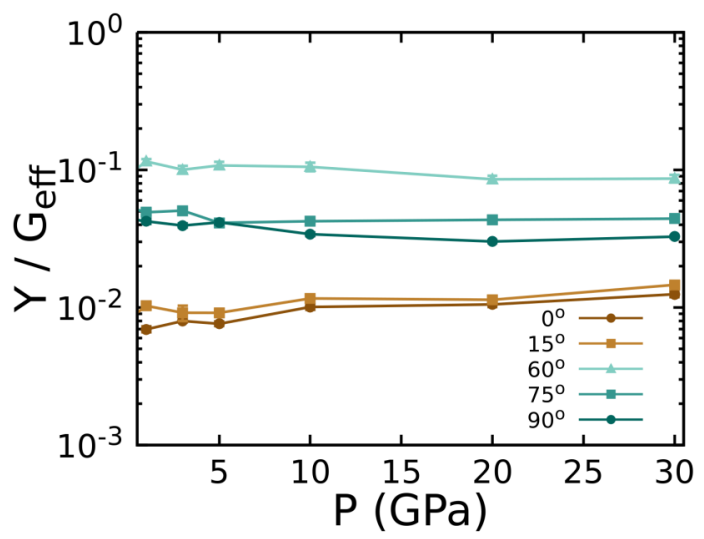

Figure 7. Steady flow stresses normalized by effective shear modulus plotted as functions of pressure. Orientations $\theta=30^{\circ}$ and $45^{\circ}$ exhibit non-steady behavior and are omitted from the plot. Note the logarithmic scale.

that this transition occurs around $15 \mathrm{GPa}$.

Bedrov et al. [43] computed a pressure-dependent polycrystalline average (isotropic) shear modulus with a similar version of the TATB FF that used rigid bonds and some subtle differences in the potential. Their values were based on a Reuss average of MD-derived elastic coefficients and varied from $1.8 \mathrm{GPa}$ at atmospheric pressure up to $\approx 8 \mathrm{GPa}$ at a pressure of $10 \mathrm{GPa}$. Their values are most similar to the effective shear modulus determined at $\theta=45^{\circ}$, but are substantially smaller than ours for the other orientations. They also reported a corresponding Voigt average of $11.5 \mathrm{GPa}$ at atmospheric pressure, which is similar in magnitude to the upper bound of the effective modulus (19.2 GPa) predicted here at $\theta=0^{\circ}$. Mathew et al. [44] showed that incorporating flexible bonds (as is the case here) leads to a modest reduction of the $C_{11}, C_{22}$, and $C_{33}$ elastic coefficients by between 8-16\%. More recently, Fan et al. [76] reported a pressure-dependent shear modulus (for shearing done on various planes and in various directions) based on the classical FF by Gee et al. [77] that exhibits a similar spread in magnitudes and degree of anisotropy as the effective modulus determined here.

Flow stresses, $Y(\theta, P)$, were extracted from the stress-strain curves for those cases exhibiting steady responses and are plotted in Figure 7. Here, the flow stress is taken as the average axial stress in $\epsilon_{z z} \in[-0.5,-0.4]$ and is normalized by the effective shear modulus $G_{\text {eff }}(\theta, P)$. Uncertainties of the flow stresses were taken as standard deviations computed over the averaging interval. Errors in $Y(\theta, P)$ and $G_{\text {eff }}(\theta, P)$ were propagated when evaluating the normalized flow stress $Y / G_{\text {eff }}$ and are on the order of the symbol size.

Most crystal orientations exhibit an approximately steady flow stress (i.e., constant axial stress) for large strains, whereas the $\theta=30^{\circ}$ and $45^{\circ}$ cases exhibit more complicated responses that are decidedly not steady. Normalized steady flow stresses for a given crystal orientation converge to a constant value with increasing pressure. There is also an apparent convergence near the bounds for $\theta$, with very similar responses for the $0^{\circ}$ 
and $15^{\circ}$ cases as well as for the $75^{\circ}$ and $90^{\circ}$ cases. The lowest normalized flow stresses are seen for axial loading that is primarily within the crystal layers $\left(\theta \leq 15^{\circ}\right)$. The results suggest that the macroscopic flow behavior of a given crystal orientation may be described using a simple scaling relation.

Nanoindentation experiments performed by Taw et al. [51] probed the response of TATB indented normal to the (001) basal plane, which best corresponds to the $\left(\theta=90^{\circ}\right.$, $P=0 \mathrm{GPa}$ ) case among our simulations. They found that the shear stress at yield varied between 0.24 and $0.48 \mathrm{GPa}$, with an average value of $0.38 \mathrm{GPa}$ across multiple experiments that is similar to our predicted steady flow stress $(0.35 \mathrm{GPa})$. Dynamic compression experiments $\left(\dot{\epsilon} \approx 10^{6} \mathrm{~s}^{-1}\right)$ performed with the molecular crystal sucrose, an inert high explosive material simulant, found a very similar strain-softening response following the the onset of yield [78]. The softening behavior was attributed to shear bands supported by thermo-viscoplastic deformation, but that appears to be distinct from the nanoscale shear banding predicted in MD simulations.

\subsection{Deformation Mechanisms}

3.2.1. Buckling/Twinning and Shear Banding Deformations in TATB crystal at large strains are bounded by two responses that predominate at the ends of the spanned crystal orientation angle $\theta \in\left[0^{\circ}, 90^{\circ}\right]$. These include the so-called buckling/twinning mechanism near $\theta=0^{\circ}$ and lattice breakdown/failure leading to regions of intense shear flow (referred to here as nanoscale shear bands) near $\theta=90^{\circ}$. A primary focus of this work is to develop quantitative measures that cleanly distinguish these features. Figure 8 shows atomically resolved snapshots of representative deformations found in the $\theta=0^{\circ}$ and $\theta=90^{\circ}$ simulations. These snapshots are oriented such that one is viewing the crystal layers edge-on along lattice vector [100].

Focusing first on the $\theta=0^{\circ}$ case, there is evidence of multiple deformation modes. The main mode is formation of buckling/twinning planes, which delineate crystalline regions with different orientations of the crystal layers. Buckling/twinning planes are especially evident through the rotational order metric (Equation 17), as molecules that fall on either side of the plane exhibit large misalignment and therefore large $\langle\psi\rangle$. Cross-inspection of the intramolecular strain energy shows that these buckling planes do not lead to significant deformations of molecular shape $\left(U_{\text {intra }} \approx 0\right)$. More substantial disorder in local packing is also apparent that is not characterized by a simple boundary plane. This manifests most intensely in comparatively small zones with large rotational disorder, such as the highlighted elliptical region. These intensely sheared regions also exhibit an increase in intramolecular strain energy, indicating that molecular shape is perturbed in addition to local crystal packing.

In comparison, the $\theta=90^{\circ}$ case exhibits a single dominant response characterized by formation of nanoscale shear bands. Molecules within the bands exhibit a loss of crystalline order, which registers as a high degree of rotational disorder. Significant strains to molecular shape are also evident, leading to large increases in intramolecular 


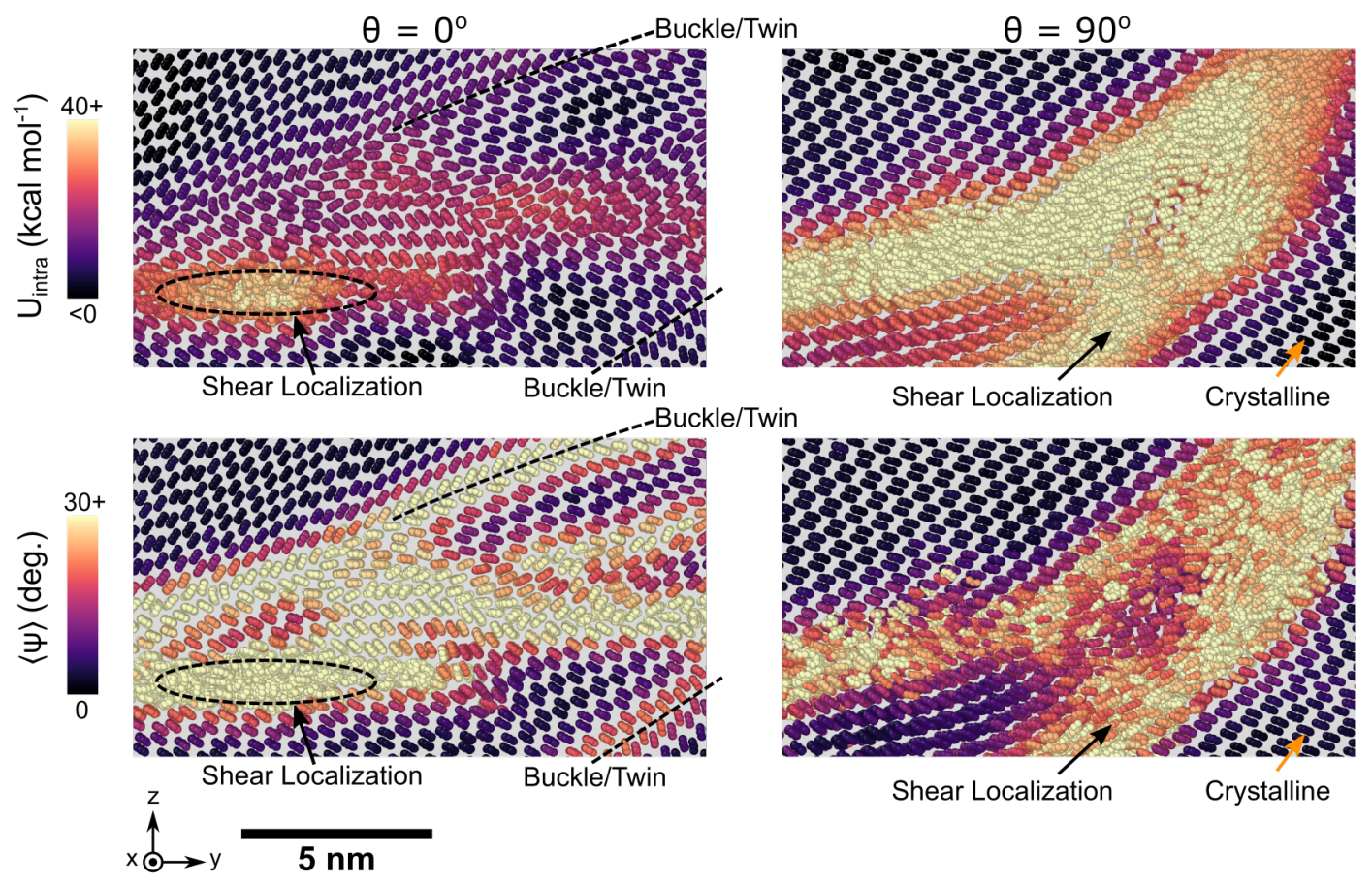

Figure 8. Renderings of representative deformation mechanisms showing the TATB $\mathrm{C}_{6}$ rings colored according to intramolecular strain energy $U_{\text {intra }}$ (Equation 14) in the first row and rotational order $\langle\psi\rangle$ (Equation 17) in the second row. The first column corresponds to state $\left(\theta=0^{\circ}, P=30 \mathrm{GPa}, \epsilon_{z z}=-0.4\right)$ and the second column to $\left(\theta=90^{\circ}, P=30 \mathrm{GPa}, \epsilon_{z z}=-0.4\right)$. Annotated features are discussed in the text.

strain energy. Regions outside the shear bands retain a high degree of crystalline order as evidenced both by visual inspection of molecule positions and numerically through small values in the rotational order metric.

Figure 9 shows a global inspection of the $\theta=0^{\circ}$ and $90^{\circ}$ cases at large strains. It is at this level that the advantages and disadvantages of the five local metrics becomes apparent. Some limitations of the simulation design can also be identified.

For $\theta=0^{\circ}$, each of the five metrics reveals a different aspect of the material response. The temperature field $T$ is largely homogeneous, indicating that there are no localized heat-producing deformation mechanisms. Intramolecular strain energy $U_{\text {intra }}$ is also small, with only nanometer-sized regions of modest excess strain. Cross-inspection of $P_{2}[\cos (\phi)]$ and rotational disorder given by $\langle\psi\rangle$ reveals the metrics are complementary, with $\langle\psi\rangle$ better revealing boundaries while $P_{2}[\cos (\phi)]$ better highlights each subgrain volume and orientation. Basing interpretations on the atomistic view in Figure 8, it is clear that $\langle\psi\rangle$ shows an extensive network of buckling/twinning planes that form loops around re-oriented crystalline domains. The shear strain $\epsilon_{\mathrm{II}}$ presents a similar view as $P_{2}[\cos (\phi)]$, but with added gradations within each crystalline domain that correspond to local sliding of molecular layers.

The $\theta=90^{\circ}$ case exhibits a number of differences on large scales that reflects the prevalence of shear banding over buckling/twinning. In particular, the temperature field 


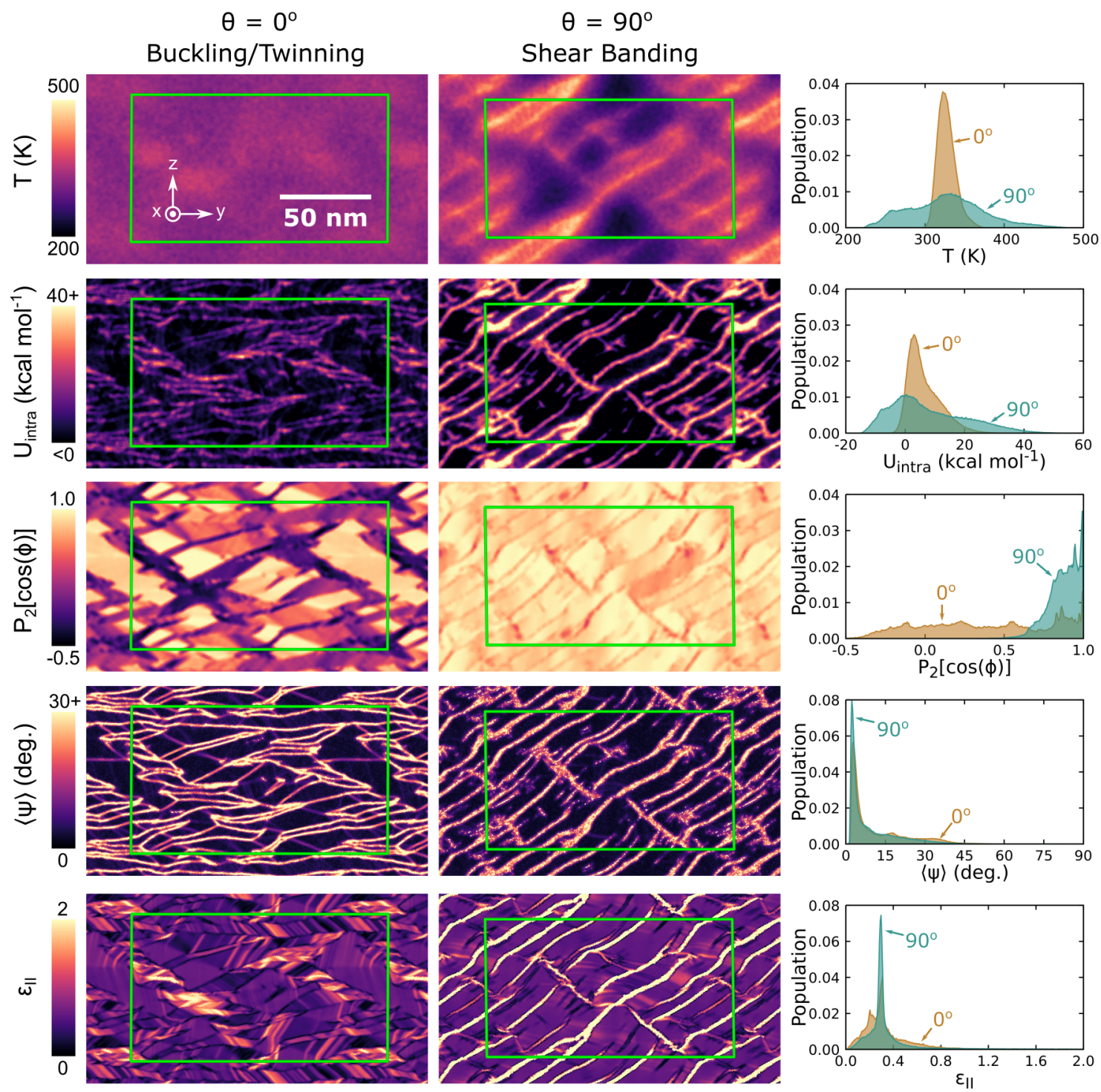

Figure 9. Snapshots for $\theta=0^{\circ}$ and $\theta=90^{\circ}\left(P=30 \mathrm{GPa}, \epsilon_{z z}=-0.4\right)$, but showing truncated replications of the entire simulation cell, which is rendered with green lines. Both configurations are colored according to the five local metrics described in Section 2.2.2. Histograms for these metrics are shown in the last column that compare the distributions for the $\theta=0^{\circ}$ and $\theta=90^{\circ}$ cases.

is inhomogenous with higher values near the shear bands revealed by the intramolecular strain energy. This is due to sustained sliding along those bands under continuous straining of the simulation cell. The thermostat balances this heating to avoid thermal runaway, but acts globally on the system leading to regions that fall below the $300 \mathrm{~K}$ target temperature. The shear bands register as regions with $P_{2}[\cos (\phi)]<1.0$ and $\langle\psi\rangle>0^{\circ}$. Similarly, the shear strain $\epsilon_{\mathrm{II}}$ quite clearly shows the shear bands. However, shear bands are regions with highly non-affine deformation, which breaks an implicit assumption in the derivation of the local deformation gradient and strain tensors.

From the perspective of quantitative feature characterization, what is perhaps most 
revealing is the comparison of the distributions for these five metrics. The temperature distribution is narrow for $0^{\circ}$ and broad for $90^{\circ}$. This distribution has a maximum above the thermostat temperature, indicating that dissipation due to sustained compressive work is not immediately quenched from the system. It is also clear that the shear bands exhibit more significant molecular distortions leading to higher values for $U_{\text {intra }}$ in the $90^{\circ}$ case. Among the structural metrics, only the $P_{2}[\cos (\phi)]$ metric leads to qualitatively different distributions for the two cases. Both buckling/twinning and shear banding exhibit practically the same distributions for rotational disorder, which complicates its use as a metric to distinguish between those two mechanisms.

Buckling/twinning has been extensively characterized at small strains by Lafourcade et al. [48] using essentially the same version of the TATB FF used here, but within a rigid molecule approximation. They found that the initial yield stress for initiating this kind of deformation exhibited a modest strain rate dependence for pure axial compression, increasing from $\approx 0.8 \mathrm{GPa}$ at $\dot{\epsilon}=10^{8} \mathrm{~s}^{-1}$ up to $\approx 1.3 \mathrm{GPa}$ at $\dot{\epsilon}=10^{10} \mathrm{~s}^{-1}$ for a crystal initially at $0 \mathrm{GPa}$. The number density of crystalline subdomains was also found to decrease with decreasing rate, yielding bigger yet fewer subdomains for a given simulation cell size. Those same authors later cast this response in terms of a phase field model that was applied to simulate weak shocks in a polycrystal [52]. It was noted that the size and shape of the simulation cell impacted the specific morphology of the buckling planes. The results obtained here with the flexible TATB FF at similar strain values $\left(\left|\epsilon_{z z}\right| \leq 0.05\right)$ are generally consistent with those of Lafourcade et al.

Reports on the response of TATB compressed approximately normal to the basal planes (near $\theta=90^{\circ}$ ) are somewhat more varied. Lafourcade et al. [48] identified nucleation of transverse dislocations that involve breaking of the hydrogen-bonded TATB layers (e.g., the $[01 \overline{1}](011)$ slip system) for pure shear along planes that are approximately $\pm 45^{\circ}$ off from the basal plane normal vector. (Loading paths required to apply this pure shear are most similar to our $\theta=90^{\circ}$ axial compression case.) As the $P \overline{1}$ TATB crystal structure has two distinct layers, these transverse dislocations involve a sterically unfavorable dilatancy with a full register shift requiring concerted breaking of two layers. Zhao et al. [49] identified the nucleation of this type of dislocation under weak shock initiation conditions $(\approx 10 \mathrm{GPa})$ for shocks normal to the basal plane in MD simulations using the flexible TATB FF, but sustained dislocation glide was hindered and prone to lattice destabilization. The predicted response for $\theta=75^{\circ}$ subject to a detonation-like shock ( $P \approx 30 \mathrm{GPa})[21]$ is essentially the same as found here during axial compression despite the (narrow) shock wave front exhibiting a rate on the order of $\dot{\epsilon}=10^{11} \mathrm{~s}^{-1}[49]$.

It is plausible, if not probable, that these observations of transverse dislocation motion and shear localization are self-consistent. The flexible molecule results of Zhao et al. show that transverse dislocation motion may serve to nucleate shear bands, but is generally unsteady. Similar pressure-dependent thresholds for dislocation lock-up followed by shear localization are predicted for some slip systems in the molecular 


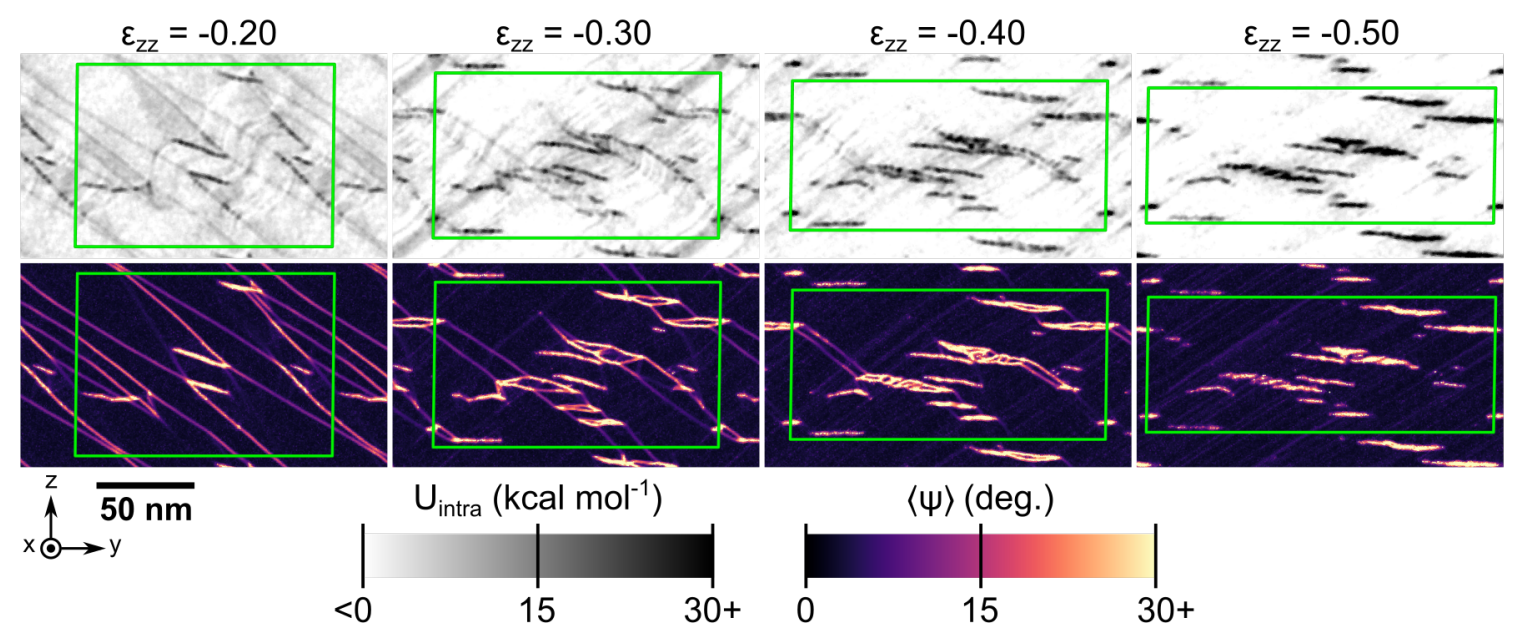

Figure 10. Time series of snapshots showing underlying structures during strain hardening for $\theta=30^{\circ}$ and $P=30 \mathrm{GPa}$. Compare to curve (i) in Figure 5.

explosive $\beta$-HMX [14], and it was also found that dislocations serve as effective nucleation points for shear bands in that system [79]. Izvekov and Rice [33] found that shear banding in coarse-grain simulations of the molecular explosive $\alpha$-RDX was sensitive to the stiffness of the effective intermolecular repulsive potential. Shear softening of this potential under shock conditions was found to depend on the degree of conformational flexibility of RDX molecules in all-atom simulations, revealing an important sensitivity in coarse-grain models that treat whole molecules as rigid bodies or point particles.

\subsubsection{Specific Mechanisms for Intermediate Orientations The previous section focused} on two of the dominant deformation mechanisms in TATB single crystal, namely the buckling/twinning mode and shear banding that arises near limiting values of orientation angle $\theta$. Here we focus on understanding a number of unusual features in the stressstrain response for intermediate angles seen in Figure 5. These include (i) the apparent strain hardening for $\theta=30^{\circ}$ at large strains, (ii) the initial stress relaxation seen for $\theta=45^{\circ}$ at small strains, and the exceptionally large strain required for the onset of significant strain softening at high (iii) and low (iv) pressure seen for $\theta=45^{\circ}$.

Figure 10 shows a series of snapshots for the $\left(\theta=30^{\circ}, P=30 \mathrm{GPa}\right)$ case at large strains where most of the other simulations predict a steady flow response. The initial response is primarily buckling/twinning, which is evidenced at $\epsilon_{z z}=-0.2$ by loops with local rotation disorder. Similar to the $\theta=0^{\circ}$ case in the previous section, the buckling planes do not exhibit significant intramolecular strain energy. As the simulation cell is continously strained, the loops migrate and interact, leaving behind defective regions within the lattice. A complementary view focused on the sub-domains would interpret those domains as growing until they meet one another; places where that meeting is incompatible form locked-in regions of defective lattice. All that remains at $\epsilon_{z z}=-0.5$ is a single-crystal volume that includes defective regions of high rotational disorder and 


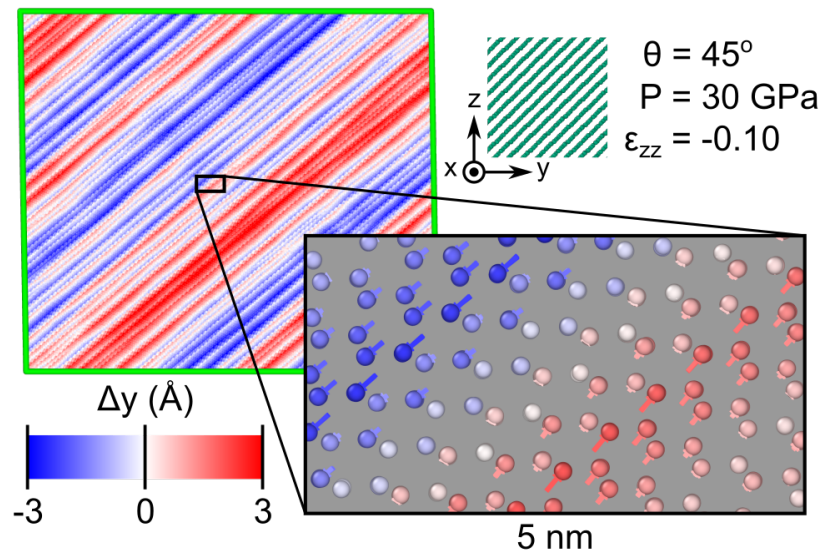

Figure 11. Layer sliding defects identified at small strains for $\theta=45^{\circ}$ and $P=30 \mathrm{GPa}$. Compare to curve (ii) in Figure 5. The inset shows molecule COMs and displacement vectors whose tails correspond to the reference positions.

intramolecular strain energy. Comparison against Figure 5 indicates that the steady increase in axial stress for $\epsilon_{z z} \leq-0.3$ coincides with the growth and interaction of subdomains. There is likely a geometric aspect of the observed hardening behavior, as the lattice is rotated by $\approx 30^{\circ}$ at larger strains. This influences the projection of stress onto the buckling/twinning systems.

The initial stress relaxation seen for $\theta=45^{\circ}$ labeled as (ii) in Figure 5 can be attributed to formation of layer-sliding defects. Figure 11 shows an example of this process at both the crystal and molecular level. Signed molecular displacements in the $y$ direction were computed relative to the reference configuration at zero strain and account for affine motion of molecules with the changing dimensions of the simulation cell. Sub-unit cell displacements of entire molecular layers develop within the basal planes, owing both to the small barriers to layer sliding [44-47] and the favorable alignment for resolved shear. Very similar defects develop for $\theta=45^{\circ}$ across the entire pressure range considered and a similar process occurs for $\theta=60^{\circ}$. Additional layer sliding is seen during compression from point (ii) up to the point of maximum axial stress at both high (iii) and low (iv) pressure. The distribution of layer sliding defects is apparently random, which indicates that this process is not a transformation to the I2/a monoclinic phase [41]. Such a transformation would be marked by displacements that repeat consistently in four-layer blocks. Magnitudes for the displacements are qualitatively consistent with the gamma surface for TATB basal glide [44,45], which exhibits multiple local minima.

Following onset of layer sliding defects for $\epsilon_{z z}<-0.1$, the $\theta=45^{\circ}$ case exhibits a pressure-dependent response at larger strains. At $30 \mathrm{GPa}$, the abrupt drop in axial stress at (iii) near $\epsilon_{z z}=-0.3$ in Figure 5 corresponds to a shear failure response producing shear bands, as is shown in Figure 12. This process initiates with lattice failure along two planes. Mass flow along the vertically oriented plane is clear from inspection of $z$ displacements. (As above, these displacements account for affine deformation of the 

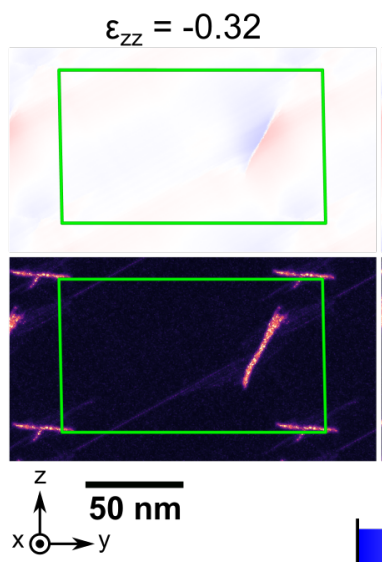

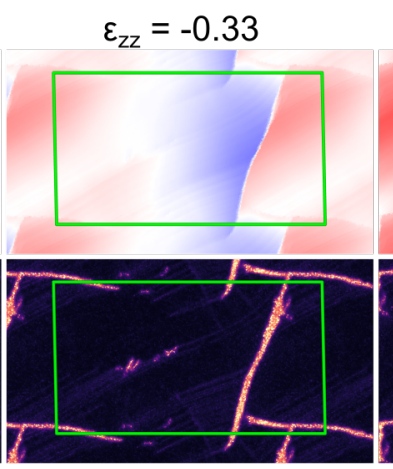

$\Delta \mathrm{z}(\mathrm{nm})$

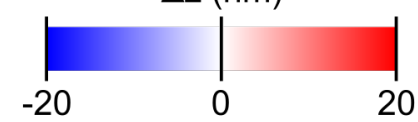

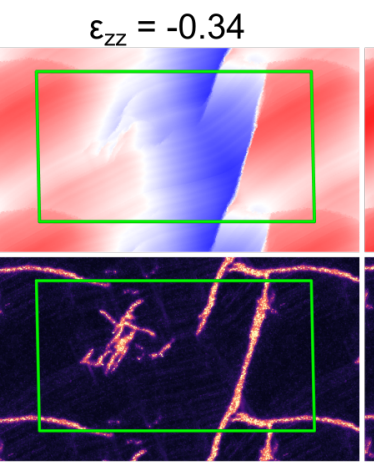

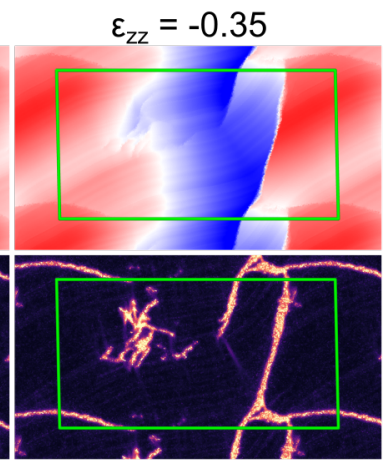

$\langle\Psi\rangle$ (deg.)

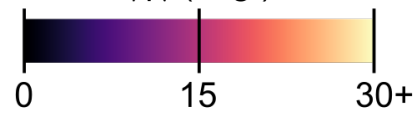

Figure 12. Time series of snapshots showing the shear failure response at large strains for the case with $\theta=45^{\circ}$ and $P=30 \mathrm{GPa}$. Color in the top row represents signed displacements of molecules in the $z$ direction relative to the reference configuration at zero strain. Compare to curve (iii) in Figure 5.

simulation cell.) Here, the left-hand side moves in $-z$ by tens of nanometers while the right-hand side moves by a similar amount in $+z$. The shear band at the interface plane has a width of $\approx 5 \mathrm{~nm}$ and contains highly disordered molecules. Once formed, the vertical band grows rapidly $\left(\Delta \epsilon_{z z}=0.01 \leftrightarrow \Delta t=10 \mathrm{ps}\right)$, ultimately spanning the simulation cell before terminating at a horizontally oriented band. The shear band dimensions and their rapid growth seen here is very similar in size and time scale to those produced under shock conditions [21,49].

A similar response is predicted for $0 \mathrm{GPa}$ at large strains (see Figure 13), which corresponds to curve (iv) in Figure 5. Here, the buildup in axial stress relieves at $\epsilon_{z z} \approx-0.25$ through formation of shear bands. However, at 0 GPa these bands serve as sites for cavitation. Multiple nanometer-sized nuclei of void space form within several shear bands at $\epsilon_{z z} \approx-0.26$ and -0.28 . These nuclei grow along the bands opening cavities measuring tens of nanometers in width, which corresponds to the axial stress release and local minimum at $\epsilon_{z z}=-0.30$. Comparison with the $30 \mathrm{GPa}$ case indicates that shear bands are initially very similar across the pressure range, but are unstable and remain cohesive only through support from the large background pressure. Inspection of the full stress tensor time history shows that the barostat maintains $P_{x x}$ and $P_{y y}$ at or slightly above $0 \mathrm{GPa}$ for the entire simulation, although this does not preclude shortlived local tensile states. The observed behavior suggests that nanoscale shear bands formed under shock wave loading may serve as sites of void nucleation and growth, which is often critical to spall response during pressure release. 

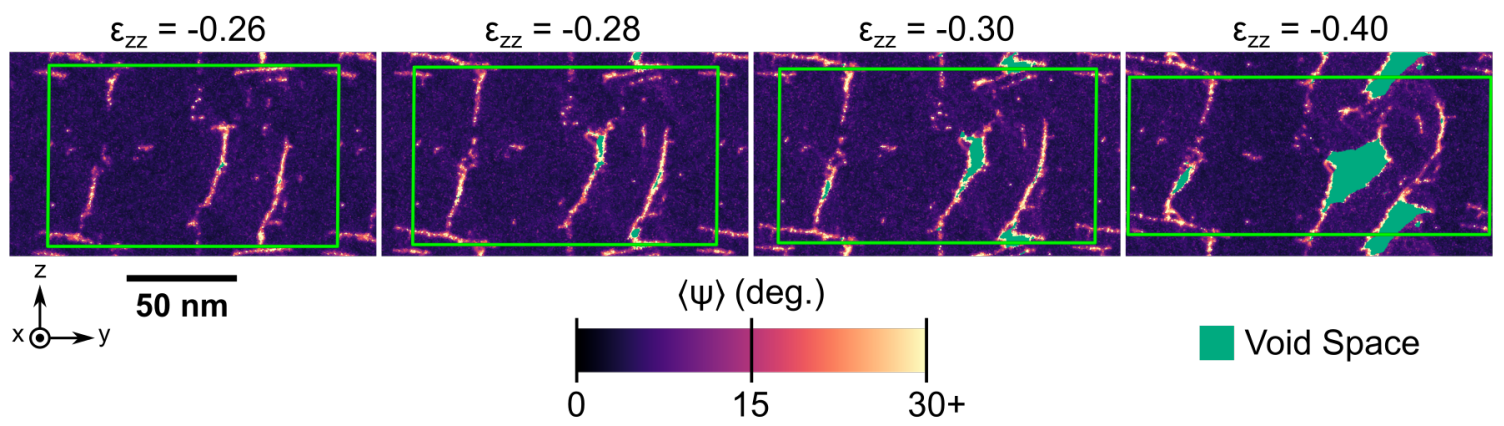

Void Space

Figure 13. Time series of snapshots showing the shear failure response at large strains for the case with $\theta=45^{\circ}$ and $P=0 \mathrm{GPa}$. Void space is colored teal. Compare to curve (iv) in Figure 5.

\subsection{Localization of Intramolecular Strain Energy}

One of the primary motivations for characterizing and quantifing subscale deformation mechanisms in TATB explosive is to identify regions with enhanced chemical reactivity. It has been shown that rotationally disordered molecules in shear bands react up to $\approx 200 \times$ faster than crystalline bulk at the same temperature under detonation conditions [21]. These bands were originally identified based on the $P_{2}[\cos (\phi)]$ metric (Equation 15). Molecular-level strains are a plausible underlying cause for this mechanochemical effect $[22,34]$ and qualitatively explain the enhanced reactivity of hot spots formed through shock-induced pore collapse [20]. TATB molecules in shear bands lose their planar shape, which increases intramolecular strain energy and may cause the observed enhancement to $\mathrm{NO}_{2}$-producing reaction channels [21] that is consistent with electronic structure predictions [80] for partial metalization with improper torsion of nitro side groups. Given the goal of identifying chemically reactive regions, the intramolecular strain energy $U_{\text {intra }}$ is the most natural variable to cast an analysis among the considered metrics. This is especially true given the ambiguity associated with interpreting the rotational order and local shear strain metrics discussed in Section 3.2. We thus examine all 49 axial compression simulations to determine which crystal orientations and pressure states give rise to shear bands or other regions of intramolecular strain localization.

Figure 14 shows assay-like snapshots of the large-strain flow state for all 49 $(\theta, P)$ cases colored according to intramolecular strain energy. This view more clearly shows separate trends with orientation and pressure. The intensity of strain energy localization increases with pressure for every orientation considered. A transition from buckling/twinning to shear banding is seen at $\theta=45^{\circ}$.

Compression nominally within the crystal layers $\left(\theta<45^{\circ}\right)$ leads to nonzero intramolecular strain values at the two highest pressures. Both the low- and highpressure response is dominated by buckling/twinning, but the low-pressure response does not exhibit regions of intense shear localization. This can be seen in the homogeneous and near-zero value of $U_{\text {intra }}$ below $10 \mathrm{GPa}$. 


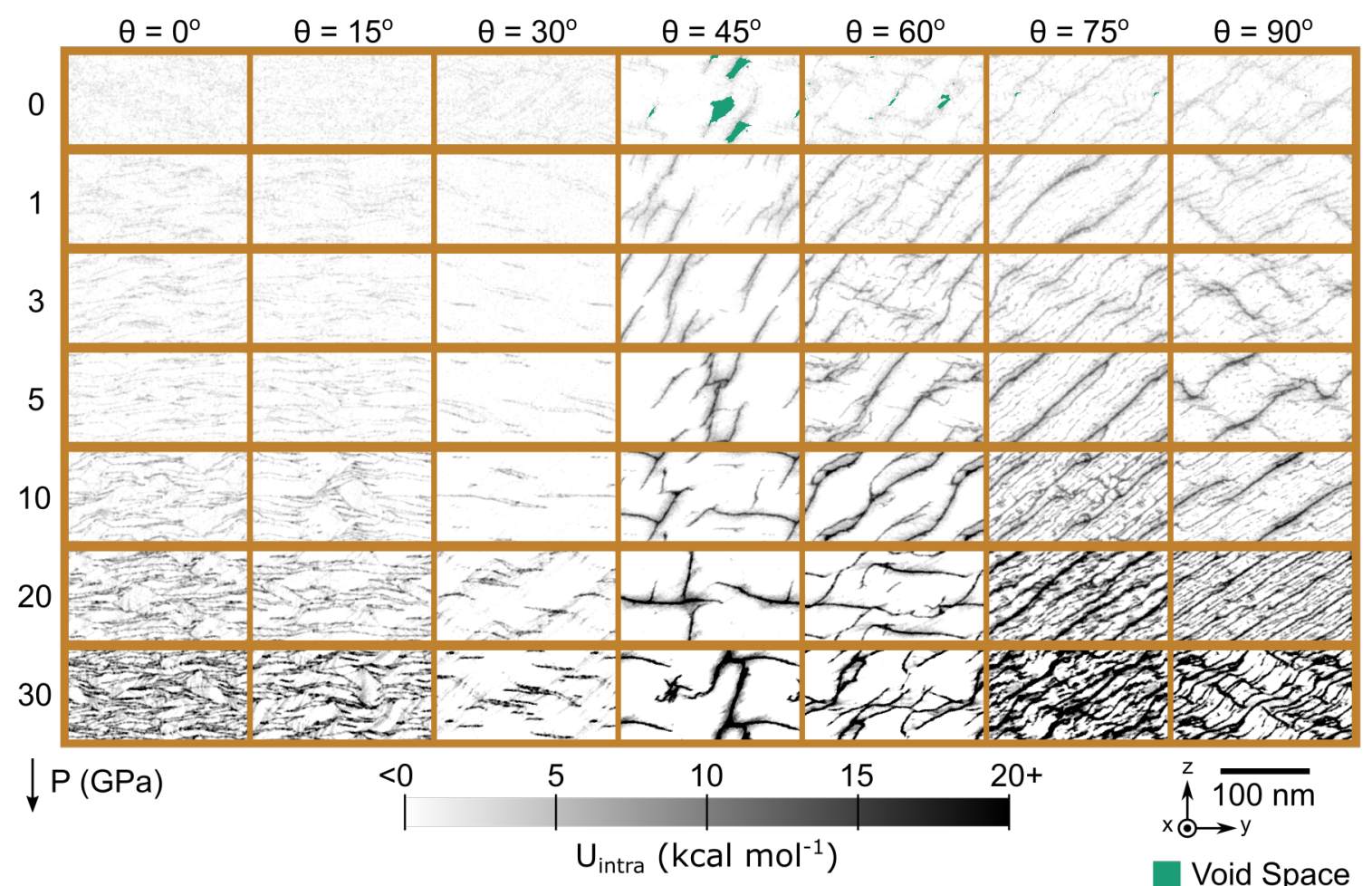

Figure 14. Snapshots showing intramolecular strain energy $U_{\text {intra }}$ for all 49 axial compression simulations taken at $\epsilon_{z z}=-0.4$, which corresponds to a steady flow state in the majority of cases. Each snapshot is a truncated replication of the simulation cell (not rendered) and periodic equivalencies can be identified within a given panel.

Cases exhibiting shear banding $\left(\theta \geq 45^{\circ}\right)$ show an increasing density of bands with increasing pressure. Individual shear bands are also somewhat wider at higher pressures. Only faint traces of bands can be seen at low pressures $(P \leq 3 \mathrm{GPa})$, indicating that pressure plays a significant role in determining whether sheared molecules retain large internal distortions and thus large intramolecular strain energy. Shear bands at low pressures are more apparent when viewed through rotational disorder $\langle\psi\rangle$, which can be seen through cross-inspection with Figure 13. Cavitation at $0 \mathrm{GPa}$ is most pronounced for $\theta=45^{\circ}$, but also manifests for $60^{\circ}$ and $75^{\circ}$. No cavities form for any orientation with $P \geq 1$ GPa.

There is a marked difference in the nature of the shear band network as $\theta$ varies from $45^{\circ}$ to $90^{\circ}$ at a constant pressure. Focusing on the $30 \mathrm{GPa}$ cases, the number density of individual band segments increases monotonically from $45^{\circ}$ to $75^{\circ}$. There is also a qualitative difference as $\theta$ varies from $60^{\circ}$ to $75^{\circ}$. The bands formed for $\theta \leq 60^{\circ}$ are comparatively sparse and few, and most notably do not coincide with the planes of maximum shear oriented at $\pm 45^{\circ}$ in the lab frame. (This trend also holds at lower pressures.) In contrast, the bands formed for $\theta \geq 75^{\circ}$ do seem preferentially aligned with these planes. While dislocations are not observed in these simulations under steady flow, differences in shear band alignment may be traceable to initial lattice destabilization 

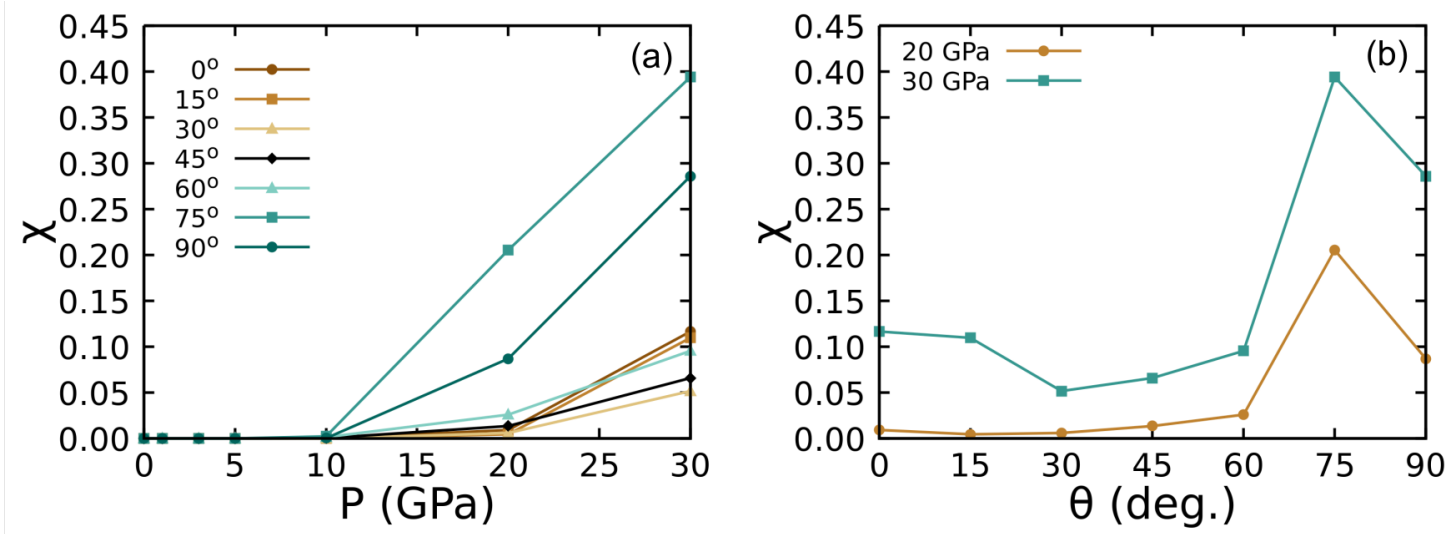

Figure 15. Mass fraction $\chi \in[0,1]$ of molecules with large intramolecular strain energy $U_{\text {intra }} \geq 10 \mathrm{kcal} \mathrm{mol}^{-1}$ plotted as a function of (a) pressure and (b) crystal orientation for the two highest pressures.

along the planes of transverse slip systems (e.g., the [011] (011) system). A more careful elucidation of shear band nucleation mechanics in TATB appears warranted in future work.

Population analysis of the intramolecular strain energy was performed to more clearly identify trends with pressure and orientation. Figure 15 shows the mass fraction $\chi$ of molecules with large $U_{\text {intra }}$ above a threshold value of $10 \mathrm{kcal} \mathrm{mol}^{-1}$. Qualitatively similar trends were found for larger thresholds, but we note that the specific value is somewhat arbitrary without a quantitative mapping between intramolecular strain energy and chemical kinetics. Inspection of the pressure plot shows that molecules only adopt highly strained conformations above $10 \mathrm{GPa}$. Even cases with shear bands $\left(\theta \geq 45^{\circ}\right)$ do not exhibit large intramolecular strains at low pressures.

Orientation dependence for the two highest pressures shows a complicated response. Maximums for both pressures are located at $\theta=75^{\circ}$, which is the same crystal orientation for which an extensive shear band network was predicted to form under strong shock conditions [21]. At $30 \mathrm{GPa}$, between $5 \%$ and $40 \%$ of molecules are in a high-energy state depending on orientation. Only the $75^{\circ}$ and $90^{\circ}$ orientations exhibit a significant population at $20 \mathrm{GPa}$. Qualitative differences in deformation mechanisms noted above are reflected in the mass-fraction plots, including the transition in shear band network character as $\theta$ increases from $60^{\circ}$ to $75^{\circ}$.

Clearly, both crystal orientation and pressure play significant roles in determining the quantity of highly strained molecules. TATB-based explosives exhibit unusual reactivity that varies parametrically with shock pressure. Continuum models capturing these features have typically relied on switching functions or entirely different parameter sets for shock initiation and steady detonation regimes [36-38]. Chemically activated shear bands have been proposed as one explanation for these differences [21] that may take the form of an additional ignition term [39]. The present results based on intramolecular strain energy are consistent with chemical activation via shear banding 
at detonation conditions, but appear to indicate that shear bands are less different from the bulk crystal at lower pressures. It is also interesting to note that buckling/twinning can lead to appreciable shear localization under large strains at detonation pressures. It may thus be reasonable to homogenize the mechanochemical consequences of the strength response in terms of a pressure-dependent ignition term that becomes active only when $P>10 \mathrm{GPa}$.

\section{Conclusions}

Mechanical strength at high strain rates plays a significant role in the localization of energy to form hot spots in high explosive materials. In the case of the layered crystalline explosive TATB (1,3,5-triamino-2,4,6-trinitrobenzene), plastic deformation through shear banding can also chemically activate the material producing an additional ignition source. Upscaling this physics to higher-level models requires determining the characteristics of TATB strength response at large strains while also quantifying the amount of chemically activated material formed by fine scale deformation modes.

We obtained predictions of TATB deformation mechanisms and stress-strain response under large strains using molecular dynamics (MD) simulations that subject single crystals to axial compression at a constant background pressure. The simulations spanned seven crystal orientations and seven pressure states. Orientations were bounded by compression exactly within and exactly normal to the crystal layers (basal plane), while the pressure ranged from atmospheric conditions up to a typical steady detonation value $(P=30 \mathrm{GPa})$.

A handful of crystal orientations exhibited strength responses that were relatively simple, involving initial yielding (at high stress in perfect crystal) and rapid strain softening supported by buckling/twinning or shear banding, settling on a steady flow stress at large strains. Effective shear moduli and flow stresses extracted from the simulations reveal a complicated dependence on crystal orientation with monotonic dependence on pressure. Steady flow states are identified for most of the crystal orientations. The ratio of flow stress to effective shear modulus for a given loading direction is found to be essentially constant in pressure, highlighting a possible route for macroscopic strength model simplification. Crystal orientations intermediate to the bounds $\left(\theta \in\left[30^{\circ}, 45^{\circ}\right]\right)$ exhibited responses that do not saturate (or become steady) for the conditions of interest, even when strained to 0.5 . These responses were traced to fine scale mechanisms.

Two previously identified $[21,48]$ deformation mechanisms are found to dominate the response at all pressures. Buckling/twinning of the molecular layers arises for compression approximately along the layers while nanoscale shear bands form for compression approximately normal to the layers. Atomic-level deformation mechanisms were analyzed using five metrics that were sensitive to different structural and molecularscale features. Some of these metrics yield quantitatively similar distributions for buckling/twinning and shear banding despite considerable differences in the two 
deformation mechanisms. A recently proposed metric [22], the intramolecular strain energy, is found to yield a clean quantitative distinction between these two mechanisms while affording a more direct connection to mechanochemistry. Large intramolecular strains manifest only above $10 \mathrm{GPa}$, and most significantly for a modest range of orientation angles that lead to extensive shear banding. All orientations generate highly strained molecules at a detonation-like pressure of $30 \mathrm{GPa}$, which reinforces shear localization as a shock-pressure-dependent ignition mode that can potentially explain TATB's unusual reactivity.

\section{Acknowledgments}

This work was performed under the auspices of the U.S. Department of Energy by Lawrence Livermore National Laboratory under Contract DE-AC52-07NA27344. It has been approved for unlimited release under document number LLNL-JRNL-827025DRAFT.

\section{References}

[1] Mader C L 1965 Phys. Fluid 81811

[2] Field J E 1992 Acc. Chem. Res. 25489

[3] Handley C A, Lambourn B D, Whitworth N J, James H R and Belfield W J 2018 Appl. Phys. Rev. 5011303

[4] Tarver C M, Chidester S K and Nichols A L 1996 J. Phys. Chem. 1005794

[5] Austin R A, Barton N R, Reaugh J E and Fried L E 2015 J. Appl. Phys. 117185902

[6] Grilli N and Koslowski M 2019 J. Appl. Phys. 126155101

[7] Springer H K, Bastea S, Nichols III A L, Tarver C M and Reaugh J E Propel. Explos. Pyrotech. 43805

[8] Rai N K and Udaykumar H S 2019 Phys. Fluids 31016103

[9] Menikoff R and Sewell T D 2002 Combust. Theory Model. 6103

[10] Lee K, Joshi K, Chaudhuri S and Stewart D S 2016 J. Chem. Phys. 144184111

[11] Barnes B C, Leiter K W, Becker R, Knap J, and Brennan J K 2017 Model. Simul. Mater. Sci. Eng. 25055006

[12] Wood M A, Kittell D E, Yarrington C D and Thompson A P 2018 Phys. Rev. B 97014109

[13] Kumar G, Van Gessel F G, Elton D C and Chung P W 2019 MRS Adv. 42191

[14] Pal A and Picu C R 2019 J. Appl. Phys. 125215111

[15] Sakano M N, Hamed A, Kober E M, Grilli N, Hamilton B W, Islam M M, Koslowski M and Strachan A 2020 J. Phys. Chem. A 1249141

[16] Holian B L, Germann T C, Maillet J B and White C T 2002 Phys. Rev. Lett. 89285501

[17] Strachan A, van Duin A C T, Chakraborty D, Dasgupta S and Goddard W A 2003 Phys. Rev. Lett. 91098301

[18] Manaa M R, Reed E J, Fried L E and Goldman N 2009 J. Am. Chem. Soc. 1315483

[19] Zybin S V, Goddard W A, Xu P, van Duin A C T and Thompson A P 2010 Appl. Phys. Lett. 96 081918

[20] Wood M A, Cherukara M J, Kober E M and Strachan A 2015 J. Phys. Chem. C 11922008

[21] Kroonblawd M P and Fried L E 2020 Phys. Rev. Lett. 124206002

[22] Hamilton B W, Kroonblawd M P, Li C and Strachan A 2021 J. Phys. Chem. Lett. 122756

[23] Zhao P, Lee S, Sewell T and Udaykumar H S Propel. Explos. Pyrotech. 45196

[24] Das P, Zhao P, Perera D, Sewell T and Udaykumar H S 2021 J. Appl. Phys. 130085901 
[25] Duarte C A, Li C, Hamilton B W, Strachan A and Koslowski M 2021 J. Appl. Phys. 129015904

[26] Winter R E, Field J E and Tabor D 1975 Proc. R. Soc. Lond. A 343399

[27] Frey R B 1981 The initiation of explosive charges by rapid shear 7th Int. Detonation Symposium

[28] Rimoli J J, Gürses E and Ortiz M 2010 Phys. Rev. B 81014112

[29] Islam M M and Strachan A 2020 J. Appl. Phys. 128065101

[30] Jaramillo E, Sewell T D and Strachan A 2007 Phys. Rev. B 76064112

[31] Cawkwell M J, Sewell T D, Zheng L and Thompson D L 2008 Phys. Rev. B 78014107

[32] Eason R M and Sewell T D 2012 J. Phys. Chem. C 1162226

[33] Izvekov S and Rice B M 2021 J. Chem. Phys. 155064503

[34] Hamilton B W, Kroonblawd M P and Strachan A 2021 The potential energy hotspot: Effects from impact velocity, defect geometry, and crystallographic orientation (Preprint 2109.12138)

[35] Cady H H and Larson A C Acta Crystallogr. 18485

[36] Lee E L and Tarver C M 1980 Phys. Fluids 232362

[37] Johnson J N, Tang P K and Forest C A 1985 J. Appl. Phys. 574323

[38] Wescott B L, Stewart D S and Davis W C 2005 J. Appl. Phys. 98053514

[39] Fried L E 2018 High explosive shock initiation model based on hot spot temperature 16th Int. Detonation Symposium

[40] Hoffman D M, Willey T M, Mitchell A R and Depiero S C 2008 J. Energ. Mater. 26139

[41] Steele B A, Clarke S M, Kroonblawd M P, Kuo I F W, Pagoria P F, Tkachev S N, Smith J S, Bastea S, Fried L E, Zaug J M, Stavrou E and Tschauner O 2019 Appl. Phys. Lett. 114191901

[42] Marshall M C, Fernandez-Panella A, Myers T W, Eggert J H, Erskine D J, Bastea S, Fried L E and Leininger L D 2020 J. Appl. Phys. 127185901

[43] Bedrov D, Borodin O, Smith G D, Sewell T D, Dattelbaum D M and Stevens L L 2009 J. Chem. Phys. 131224703

[44] Mathew N and Sewell T D 2015 Phil. Mag. 95424

[45] Lafourcade P, Denoual C and Maillet J B 2017 J. Phys. Chem. C 1217442

[46] Kroonblawd M P and Sewell T D 2014 J. Chem. Phys. 141184501

[47] Steele B A, Stavrou E, Prakapenka V B, Kroonblawd M P and Kuo I F W 2020 J. Phys. Chem. A 12410580

[48] Lafourcade P, Denoual C and Maillet J B 2018 J. Phys. Chem. C 12214954

[49] Zhao P, Kroonblawd M, Mathew N and Sewell $T 2021$ ChemRxiv URL https://chemrxiv.org/engage/chemrxiv/article-details/60c73cc0842e65d907db1680

[50] Mathew N and Sewell T D 2016 J. Phys. Chem. C 1208266

[51] Taw M R, Yeager J D, Hooks D E, Carvajal T M and Bahr D F 2017 J. Mater. Res. 322728

[52] Lafourcade P, Denoual C and Maillet J B 2019 Phys. Rev. Mat. 3053610

[53] Honeycutt J D and Andersen H C 1987 J. Phys. Chem. 914950

[54] Ackland G J and Jones A P 2006 Phys. Rev. B 73054104

[55] Larsen P M, Schmidt S and Schiøtz J 2016 Model. Simul. Mater. Sci. Eng. 24055007

[56] Falk M L and Langer J S 1998 Phys. Rev. E 57(6) 7192

[57] Shimizu F, Ogata S and Li J 2007 Mat. Trans. 482923

[58] Kroonblawd M P, Mathew N, Jiang S and Sewell T D 2016 Comput. Phys. Commun. 207232

[59] Plimpton S 1995 J. Comput. Phys. 1171

[60] Kroonblawd M P and Sewell T D 2013 J. Chem. Phys. 139074503

[61] Mathew N, Sewell T D and Thompson D L 2015 J. Chem. Phys. 143094706

[62] Wolf D, Keblinski P, Phillpot S R and Eggebrecht J 1999 J. Chem. Phys. 1108254

[63] Nosé S 1984 J. Chem. Phys. 81511

[64] Hoover W G 1985 Phys. Rev. A 311695

[65] Martyna G J, Tobias D J and Klein M L 1994 J. Chem. Phys. 1014177

[66] Stukowski A Model. Simul. Mater. Sci. Eng. 18015012

[67] Zepeda-Ruiz L A, Stukowski A, Oppelstrup T and Bulatov V V 2017 Nature 550492

[68] Plisson T, Pineau N, Weck G, Bruneton E, Guignot N and Loubeyre P 2017 J. Appl. Phys. 122 
235901

[69] Zaug J M, Stavrou E, Wixon R R, Bastea S and Prakapenka V 2017 Isothermal and Shock Hugoniot EOSs of TATB: A Combined Experimental and Theoretical Study 16th Int. Detonation Symposium

[70] Adamo C and Barone V 1999 J. Chem. Phys. 1106158

[71] Grimme S J. Comp. Chem. 271787

[72] Stevens L L, Velisavljevic N, Hooks D E and Dattelbaum D M Propel. Explos. Pyrotech. 33286

[73] Crowhurst J C, Armstrong M R, Knight K B, Zaug J M and Behymer E M 2011 Phys. Rev. Lett. $\mathbf{1 0 7}(14) 144302$

[74] Chen K and Schweizer K S 2011 Macromol. 443988

[75] Willey T M, vanBuuren T, Lee J R, Overturf G E, Kinney J H, Handly J, Weeks B L and Ilavsky J Propel. Explos. Pyrotech. 31466

[76] Fan H, Long Y, Ding L, Chen J and Nie F D 2017 Comp. Mat. Sci. 131321

[77] Gee R H, Roszak S, Balasubramanian K and Fried L E 2004 J. Chem. Phys. 1207059

[78] Malhotra P, Jiao T, Henann D L, Clifton R J and Guduru P R 2021 J. Mech. Phys. Solids 104624

[79] Khan M and Picu C R 2020 J. Appl. Phys. 128105902

[80] Riad Manaa M 2003 Appl. Phys. Lett. 831352 